\title{
A Note on the Second Order of Accuracy Stable Difference Schemes for the Nonlocal Boundary Value Hyperbolic Problem
}

\author{
Allaberen Ashyralyev ${ }^{1}$ and Ozgur Yildirim ${ }^{2}$ \\ ${ }^{1}$ Department of Mathematics, Fatih University, Buyukcekmece 34500, Istanbul, Turkey \\ ${ }^{2}$ Department of Mathematics, Yildiz Technical University, Esenler 34210, Istanbul, Turkey \\ Correspondence should be addressed to Ozgur Yildirim, ozgury@yildiz.edu.tr
}

Received 23 March 2012; Accepted 11 June 2012

Academic Editor: Sergey Piskarev

Copyright (C) 2012 A. Ashyralyev and O. Yildirim. This is an open access article distributed under the Creative Commons Attribution License, which permits unrestricted use, distribution, and reproduction in any medium, provided the original work is properly cited.

\begin{abstract}
The second order of accuracy absolutely stable difference schemes are presented for the nonlocal boundary value hyperbolic problem for the differential equations in a Hilbert space $H$ with the self-adjoint positive definite operator $A$. The stability estimates for the solutions of these difference schemes are established. In practice, one-dimensional hyperbolic equation with nonlocal boundary conditions and multidimensional hyperbolic equation with Dirichlet conditions are considered. The stability estimates for the solutions of these difference schemes for the nonlocal boundary value hyperbolic problem are established. Finally, a numerical method proposed and numerical experiments, analysis of the errors, and related execution times are presented in order to verify theoretical statements.
\end{abstract}

\section{Introduction}

Hyperbolic partial differential equations play an important role in many branches of science and engineering and can be used to describe a wide variety of phenomena such as acoustics, electromagnetics, hydrodynamics, elasticity, fluid mechanics, and other areas of physics (see [1-5] and the references given therein).

While applying mathematical modelling to several phenomena of physics, biology, and ecology, there often arise problems with nonclassical boundary conditions, which the values of unknown function on the boundary are connected with inside of the given domain. Such type of boundary conditions are called nonlocal boundary conditions. Over the last decades, boundary value problems with nonlocal boundary conditions have become a rapidly growing area of research (see, e.g., [6-16] and the references given therein). 
In the present work, we consider the nonlocal boundary value problem

$$
\begin{aligned}
& \frac{d^{2} u(t)}{d t^{2}}+A u(t)=f(t) \quad(0 \leq t \leq 1), \\
& u(0)=\sum_{j=1}^{n} \alpha_{j} u\left(\lambda_{j}\right)+\varphi, \quad u_{t}(0)=\sum_{j=1}^{n} \beta_{j} u_{t}\left(\lambda_{j}\right)+\psi, \\
& 0<\lambda_{1}<\lambda_{2}<\cdots<\lambda_{n} \leq 1,
\end{aligned}
$$

where $A$ is a self-adjoint positive definite operator in a Hilbert space $H$.

A function $u(t)$ is called a solution of the problem (1.1), if the following conditions are satisfied:

(i) $u(t)$ is twice continuously differentiable on the segment $[0,1]$. The derivatives at the endpoints of the segment are understood as the appropriate unilateral derivatives.

(ii) The element $u(t)$ belongs to $D(A)$, independent of $t$, and dense in $H$ for all $t \in[0,1]$ and the function $A u(t)$ is continuous on the segment $[0,1]$.

(iii) $u(t)$ satisfies the equation and nonlocal boundary conditions (1.1).

In the paper of [8], the following theorem on the stability estimates for the solution of the nonlocal boundary value problem (1.1) was proved.

Theorem 1.1. Suppose that $\varphi \in D(A), \psi \in D\left(A^{1 / 2}\right)$, and $f(t)$ is a continuously differentiable function on $[0,1]$ and the assumption

$$
\sum_{k=1}^{n}\left|\alpha_{k}+\beta_{k}\right|+\sum_{m=1}^{n}\left|\alpha_{m}\right| \sum_{\substack{k=1 \\ k \neq m}}^{n}\left|\beta_{k}\right|<\left|1+\sum_{k=1}^{n} \alpha_{k} \beta_{k}\right|
$$

holds. Then, there is a unique solution of problem (1.1) and the stability inequalities

$$
\begin{gathered}
\max _{0 \leq t \leq 1}\|u(t)\|_{H} \leq M\left[\|\varphi\|_{H}+\left\|A^{-1 / 2} \psi\right\|_{H}+\max _{0 \leq t \leq 1}\left\|A^{-1 / 2} f(t)\right\|_{H}\right], \\
\max _{0 \leq t \leq 1}\left\|A^{1 / 2} u(t)\right\|_{H} \leq M\left[\left\|A^{1 / 2} \varphi\right\|_{H}+\|\varphi\|_{H}+\max _{0 \leq t \leq 1}\|f(t)\|_{H}\right], \\
\max _{0 \leq t \leq 1}\left\|\frac{d^{2} u(t)}{d t^{2}}\right\|_{H}+\max _{0 \leq t \leq 1}\|A u(t)\|_{H} \leq M\left[\|A \varphi\|_{H}+\left\|A^{1 / 2} \psi\right\|_{H}+\|f(0)\|_{H}+\int_{0}^{1}\left\|f^{\prime}(t)\right\|_{H} d t\right]
\end{gathered}
$$

hold, where $M$ does not depend on $\varphi, \psi$, and $f(t), t \in[0,1]$.

Moreover, the first order of accuracy difference scheme

$$
\begin{aligned}
& \tau^{-2}\left(u_{k+1}-2 u_{k}+u_{k-1}\right)+A u_{k+1}=f_{k}, \quad f_{k}=f\left(t_{k}\right), \\
& t_{k}=k \tau, \quad 1 \leq k \leq N-1, N \tau=1,
\end{aligned}
$$




$$
\begin{aligned}
& u_{0}=\sum_{r=1}^{n} \alpha_{r} u_{\left[\lambda_{r} / \tau\right]}+\varphi, \\
& \tau^{-1}\left(u_{1}-u_{0}\right)=\sum_{r=1}^{n} \beta_{r}\left(u_{\left[\lambda_{r} / \tau\right]+1}-u_{\left[\lambda_{r} / \tau\right]}\right) \frac{1}{\tau}+\psi,
\end{aligned}
$$

for the approximate solution of problem (1.1) was presented. The stability estimates for the solution of this difference scheme, under the assumption

$$
\sum_{k=1}^{n}\left|\alpha_{k}\right|+\sum_{k=1}^{n}\left|\beta_{k}\right|+\sum_{k=1}^{n}\left|\alpha_{k}\right| \sum_{k=1}^{n}\left|\beta_{k}\right|<1
$$

were established.

In the development of numerical techniques for solving PDEs, the stability has been an important research topic (see [6-31]). A large cycle of works on difference schemes for hyperbolic partial differential equations, in which stability was established under the assumption that the magnitude of the grid steps $\tau$ and $h$ with respect to the time and space variables, are connected. In abstract terms, this particularly means that $\tau\left\|A_{h}\right\| \rightarrow 0$ when $\tau \rightarrow 0$.

We are interested in studying the high order of accuracy difference schemes for hyperbolic PDEs, in which stability is established without any assumption with respect to the grid steps $\tau$ and $h$. Particularly, a convenient model for analyzing the stability is provided by a proper unconditionally absolutely stable difference scheme with an unbounded operator.

In the present paper, the second order of accuracy unconditionally stable difference schemes for approximately solving boundary value problem (1.1) is presented. The stability estimates for the solutions of these difference schemes and their first and second order difference derivatives are established. This operator approach permits one to obtain the stability estimates for the solutions of difference schemes of nonlocal boundary value problems, for one-dimensional hyperbolic equation with nonlocal boundary conditions in space variable and multidimensional hyperbolic equation with Dirichlet condition in space variables.

Some results of this paper without proof were presented in [7].

Note that nonlocal boundary value problems for parabolic equations, elliptic equations, and equations of mixed types have been studied extensively by many scientists (see, e.g., [11-16, 20-24, 32-38] and the references therein).

\section{The Second Order of Accuracy Difference Scheme Generated by $A^{2}$}

Throughout this paper for simplicity $\lambda_{1}>2 \tau$ and $\lambda_{n}<1$ will be considered. Let us associate boundary value problem (1.1) with the second order of accuracy difference scheme

$$
\begin{aligned}
& \tau^{-2}\left(u_{k+1}-2 u_{k}+u_{k-1}\right)+A u_{k}+\frac{\tau^{2}}{4} A^{2} u_{k+1}=f_{k} \\
& f_{k}=f\left(t_{k}\right), \quad t_{k}=k \tau, 1 \leq k \leq N-1, N \tau=1
\end{aligned}
$$




$$
\begin{aligned}
& u_{0}=\sum_{m=1}^{n} \alpha_{m}\left\{u_{\left[\lambda_{m} / \tau\right]}+\tau^{-1}\left(u_{\left[\lambda_{m} / \tau\right]}-u_{\left[\lambda_{m} / \tau\right]-1}\right) \times\left(\lambda_{m}-\left[\frac{\lambda_{m}}{\tau}\right] \tau\right)\right\}+\varphi, \\
& \left(I+\frac{\tau^{2} A}{2}\right) \tau^{-1}\left(u_{1}-u_{0}\right)-\frac{\tau}{2}\left(f_{0}-A u_{0}\right) \\
& =\sum_{k=1}^{n} \beta_{k}\left\{\tau^{-1}\left(u_{\left[\lambda_{k} / \tau\right]}-u_{\left[\lambda_{k} / \tau\right]-1}\right)+\left(\frac{\tau}{2}+\left(\lambda_{k}-\left[\frac{\lambda_{k}}{\tau}\right] \tau\right)\right) \times\left(f_{\left[\lambda_{k} / \tau\right]}-A u_{\left[\lambda_{k} / \tau\right]}\right)\right\}+\psi, \\
& f_{0}=f(0) \text {. }
\end{aligned}
$$

A study of discretization, over time only, of the nonlocal boundary value problem also permits one to include general difference schemes in applications, if the differential operator in space variables $A$ is replaced by the difference operator $A_{h}$ that act in the Hilbert space and are uniformly self-adjoint positive definite in $h$ for $0<h \leq h_{0}$.

In general, we have not been able to obtain the stability estimates for the solution of difference scheme (2.1) under assumption (1.5). Note that the stability of solution of difference scheme (2.1) will be obtained under the strong assumption

$$
\begin{gathered}
\sum_{k=1}^{n}\left|\alpha_{k}\right|\left(1+\left|\lambda_{k}-\left[\frac{\lambda_{k}}{\tau}\right] \tau\right|\right)+\sum_{k=1}^{n}\left|\beta_{k}\right|\left(1+\left|\lambda_{k}-\left[\frac{\lambda_{k}}{\tau}\right] \tau\right|\right) \\
+\sum_{k=1}^{n}\left|\alpha_{k}\right| \sum_{k=1}^{n}\left|\beta_{k}\right|\left(1+\left|\lambda_{k}-\left[\frac{\lambda_{k}}{\tau}\right] \tau\right|\right) \\
+\sum_{k=1}^{n}\left|\alpha_{k}\right|\left|\lambda_{k}-\left[\frac{\lambda_{k}}{\tau}\right] \tau\right| \sum_{k=1}^{n}\left|\beta_{k}\right|\left|\lambda_{k}-\left[\frac{\lambda_{k}}{\tau}\right] \tau\right|<1 .
\end{gathered}
$$

Now, let us give some lemmas that will be needed in the sequel.

Lemma 2.1. The following estimates hold:

$$
\begin{aligned}
& \|R\|_{H \mapsto H} \leq 1, \quad\|\tilde{R}\|_{H \mapsto H} \leq 1, \\
& \left\|\tilde{R}^{-1} R\right\|_{H \mapsto H} \leq 1, \quad\left\|R^{-1} \tilde{R}\right\|_{H \mapsto H} \leq 1, \\
& \left\|\tau A^{1 / 2} R\right\|_{H \mapsto H} \leq 1, \quad\left\|\tau A^{1 / 2} \tilde{R}\right\|_{H \mapsto H} \leq 1 .
\end{aligned}
$$

Here, $H$ is the Hilbert space, $R=\left(I+i \tau A^{1 / 2}-\left(\tau^{2} / 2\right) A\right)^{-1}$, and $\widetilde{R}=\left(I-i \tau A^{1 / 2}-\left(\tau^{2} / 2\right) A\right)^{-1}$.

Lemma 2.2. Suppose that assumption (2.2) holds. Denote

$$
\begin{aligned}
B_{\tau}= & \sum_{m=1}^{n} \alpha_{m}\left(I+\frac{\tau^{2} A}{2}\right)^{-1}\left[\frac{R^{\left[\lambda_{m} / \tau\right]-1}\left(I-i \tau A^{1 / 2}\right)+\widetilde{R}^{\left[\lambda_{m} / \tau\right]-1}\left(I+i \tau A^{1 / 2}\right)}{2}\right] \\
& +\sum_{k=1}^{n} \beta_{k}\left(I+\frac{\tau^{2} A}{2}\right)^{-1}\left[\frac{\widetilde{R}^{\left[\lambda_{k} / \tau\right]-1} R^{-1}+R^{\left[\lambda_{k} / \tau\right]-1} \widetilde{R}^{-1}}{2}\right]
\end{aligned}
$$




$$
\begin{aligned}
& -\frac{1}{2} \sum_{m=1}^{n} \sum_{k=1}^{n} \alpha_{m} \beta_{k}\left(I+\frac{\tau^{2} A}{2}\right)^{-1}\left[R^{\left[\lambda_{m} / \tau\right]-1} \tilde{R}^{\left[\lambda_{k} / \tau\right]-1}+\tilde{R}^{\left[\lambda_{m} / \tau\right]-1} R^{\left[\lambda_{k} / \tau\right]-1}\right] \\
& +\sum_{m=1}^{n} \alpha_{m}\left(\lambda_{m}-\left[\frac{\lambda_{m}}{\tau}\right] \tau\right)\left(i A^{1 / 2}\right)\left(I+\frac{\tau^{2} A}{2}\right)^{-1} \\
& \times\left[\frac{\widetilde{R}^{\left[\lambda_{m} / \tau\right]-1}\left(I-(i \tau / 2) A^{1 / 2}\right)\left(I+i \tau A^{1 / 2}\right)-R^{\left[\lambda_{m} / \tau\right]-1}\left(I+(i \tau / 2) A^{1 / 2}\right)\left(I-i \tau A^{1 / 2}\right)}{2}\right] \\
& +\sum_{k=1}^{n} \beta_{k}\left(\lambda_{k}-\left[\frac{\lambda_{k}}{\tau}\right] \tau\right)\left(i A^{1 / 2}\right)\left(I+\frac{\tau^{2} A}{2}\right)^{-1}\left[\frac{\widetilde{R}^{\left[\lambda_{k} / \tau\right]-1} R^{-1}-R^{\left[\lambda_{k} / \tau\right]-1} \widetilde{R}^{-1}}{2}\right] \\
& +\frac{1}{4} \sum_{m=1}^{n} \sum_{k=1}^{n} \alpha_{m} \beta_{k}\left(\lambda_{m}-\left[\frac{\lambda_{m}}{\tau}\right] \tau\right)\left(I+\frac{\tau^{2} A}{2}\right)^{-2} \\
& \times i A^{1 / 2}\left[R^{\left[\lambda_{m} / \tau\right]-1} \tilde{R}^{\left[\lambda_{k} / \tau\right]-1}\left(I+\frac{i \tau}{2} A^{1 / 2}\right)-\widetilde{R}^{\left[\lambda_{m} / \tau\right]-1} R^{\left[\lambda_{k} / \tau\right]-1}\left(I-\frac{i \tau}{2} A^{1 / 2}\right)\right] \\
& -\frac{1}{2} \sum_{m=1}^{n} \sum_{k=1}^{n} \alpha_{m} \beta_{k}\left(\lambda_{k}-\left[\frac{\lambda_{k}}{\tau}\right] \tau\right)\left(i A^{1 / 2}\right)\left(I+\frac{\tau^{2} A}{2}\right)^{-1} \\
& \times\left[R^{\left[\lambda_{m} / \tau\right]-1} \widetilde{R}^{\left[\lambda_{k} / \tau\right]-1}-\widetilde{R}^{\left[\lambda_{m} / \tau\right]-1} R^{\left[\lambda_{k} / \tau\right]-1}\right] \\
& -\frac{1}{2} \sum_{m=1}^{n} \sum_{k=1}^{n} \alpha_{m} \beta_{k}\left(\lambda_{m}-\left[\frac{\lambda_{m}}{\tau}\right] \tau\right)\left(\lambda_{k}-\left[\frac{\lambda_{k}}{\tau}\right] \tau\right) A\left(I+\frac{\tau^{2} A}{2}\right)^{-1} \\
& \times\left[\tilde{R}^{\left[\lambda_{m} / \tau\right]-1} R^{\left[\lambda_{k} / \tau\right]-1}\left(I-\frac{i \tau}{2} A^{1 / 2}\right)+R^{\left[\lambda_{m} / \tau\right]-1} \tilde{R}^{\left[\lambda_{k} / \tau\right]-1}\left(I+\frac{i \tau}{2} A^{1 / 2}\right)\right] \\
&
\end{aligned}
$$

Then, the operator $I-B_{\tau}$ has an inverse

$$
T_{\tau}=\left(I-B_{\tau}\right)^{-1},
$$

and the following estimate holds:

$$
\left\|T_{\tau}\right\|_{H \mapsto H} \leq M .
$$

Proof. The proof of estimate (2.6) is based on the estimate

$$
\begin{aligned}
\left\|I-B_{\tau}\right\|_{H \mapsto H} \geq & -\sum_{k=1}^{n}\left|\alpha_{k}\right|\left(1+\left|\lambda_{k}-\left[\frac{\lambda_{k}}{\tau}\right] \tau\right|\right)-\sum_{k=1}^{n}\left|\beta_{k}\right|\left(1+\left|\lambda_{k}-\left[\frac{\lambda_{k}}{\tau}\right] \tau\right|\right) \\
& -\sum_{k=1}^{n}\left|\alpha_{k}\right| \sum_{k=1}^{n}\left|\beta_{k}\right|\left(1+\left|\lambda_{k}-\left[\frac{\lambda_{k}}{\tau}\right] \tau\right|\right) \\
& -\sum_{k=1}^{n}\left|\alpha_{k}\right|\left|\lambda_{k}-\left[\frac{\lambda_{k}}{\tau}\right] \tau\right| \sum_{k=1}^{n}\left|\beta_{k}\right|\left|\lambda_{k}-\left[\frac{\lambda_{k}}{\tau}\right] \tau\right| .
\end{aligned}
$$

Estimate (2.7) follows from the triangle inequality and estimate (2.3). Lemma 2.2 is proved. 
Now, we will obtain the formula for the solution of problem (2.1). It is easy to show that (see, e.g., [18]) there is unique solution of the problem

$$
\begin{aligned}
& \tau^{-2}\left(u_{k+1}-2 u_{k}+u_{k-1}\right)+A u_{k}+\frac{\tau^{2}}{4} A^{2} u_{k+1}=f_{k}, \\
& f_{k}=f\left(t_{k}\right), \quad t_{k}=k \tau, 1 \leq k \leq N-1, N \tau=1, \\
& \left(I+\frac{\tau^{2} A}{2}\right) \tau^{-1}\left(u_{1}-u_{0}\right)-\frac{\tau}{2}\left(f_{0}-A u_{0}\right)=\omega, \quad f_{0}=f(0), \quad u_{0}=\mu,
\end{aligned}
$$

and the following formula holds:

$$
\begin{gathered}
u_{0}=\mu, \quad u_{1}=\left(I+\frac{\tau^{2} A}{2}\right)^{-1}\left(\mu+\tau \omega+\frac{\tau^{2}}{2} f_{0}\right], \\
u_{k}=\left(I+\frac{\tau^{2} A}{2}\right)^{-1}\left(\frac{R^{k-1}\left(I-i \tau A^{1 / 2}\right)+\widetilde{R}^{k-1}\left(I+i \tau A^{1 / 2}\right)}{2}\right) \mu \\
+\left(I+\frac{\tau^{2} A}{2}\right)^{-1}\left(i A^{1 / 2}\right)^{-1}\left(\frac{\widetilde{R}^{k-1} R^{-1}-R^{k-1} \widetilde{R}^{-1}}{2}\right)\left(\omega+\frac{\tau}{2} f_{0}\right) \\
-\sum_{s=1}^{k-1} \frac{\tau}{2 i} A^{-1 / 2}\left(R^{k-s}-\widetilde{R}^{k-s}\right) f_{s,} \quad 2 \leq k \leq N .
\end{gathered}
$$

Applying formula (2.9) and the nonlocal boundary conditions in problem (2.1), we get

$$
\begin{aligned}
& \mu=T_{\tau}\left\{\left[\sum_{m=1}^{n} \alpha_{m} \frac{\tau}{2} f_{0}\right.\right. \\
& \times\left(\left(I+\frac{\tau^{2} A}{2}\right)^{-1}\left(i A^{1 / 2}\right)^{-1} \times \frac{\tilde{R}^{\left[\lambda_{m} / \tau\right]-1} R^{-1}-R^{\left[\lambda_{m} / \tau\right]-1} \tilde{R}^{-1}}{2}\right. \\
& +\sum_{m=1}^{n} \alpha_{m}\left(\lambda_{m}-\left[\frac{\lambda_{m}}{\tau}\right] \tau\right)\left(I+\frac{\tau^{2} A}{2}\right)^{-1} \\
& \left.\times\left(\frac{\widetilde{R}^{\left[\lambda_{m} / \tau\right]-1} R^{-1}\left(I-(i \tau / 2) A^{1 / 2}\right)+R^{\left[\lambda_{m} / \tau\right]-1} \widetilde{R}^{-1}\left(I+(i \tau / 2) A^{1 / 2}\right)}{2}\right)\right) \\
& -\left(\sum_{m=1}^{n} \alpha_{m} \sum_{s=1}^{\left[\lambda_{m} / \tau\right]_{-1}} \frac{\tau}{2 i} A^{-1 / 2}\left(R^{\left[\lambda_{m} / \tau\right]-\mathrm{s}}-\widetilde{R}^{\left[\lambda_{m} / \tau\right]-\mathrm{s}}\right) f_{s}-\sum_{m=1}^{n} \alpha_{m}\left(\lambda_{m}-\left[\frac{\lambda_{m}}{\tau}\right] \tau\right)\right. \\
& \times \sum_{s=1}^{\left[\lambda_{m} / \tau\right]} \frac{\tau}{2 i} A^{1 / 2}\left(i A^{-1 / 2}\right)\left(R^{\left[\lambda_{m} / \tau\right]-\mathrm{s}}\left(I-\frac{i \tau}{2} A^{1 / 2}\right)+\tilde{R}^{\left[\lambda_{m} / \tau\right]-\mathrm{s}}\left(I-\frac{i \tau}{2} A^{1 / 2}\right)\right) f_{s} \\
& \left.\left.+\sum_{m=1}^{n} \alpha_{m} \tau\left(\lambda_{m}-\left[\frac{\lambda_{m}}{\tau}\right] \tau\right) R \widetilde{R} f_{\left[\lambda_{m} / \tau\right]-1}-\varphi\right)\right]
\end{aligned}
$$




$$
\begin{aligned}
& \times\left[I+\sum_{k=1}^{n} \beta_{k}\left(\frac{\tau}{2}+\left(\lambda_{k}-\left[\frac{\lambda_{k}}{\tau}\right] \tau\right)\right) A\left(I+\frac{\tau^{2} A}{2}\right)^{-1}\left(i A^{1 / 2}\right)^{-1}\right. \\
& \times\left(\frac{\widetilde{R}^{\left[\lambda_{k} / \tau\right]-1} R^{-1}-R^{\left[\lambda_{k} / \tau\right]-1} \widetilde{R}^{-1}}{2}\right)-\sum_{m=1}^{n} \alpha_{m}\left(\lambda_{m}-\left[\frac{\lambda_{m}}{\tau}\right] \tau\right)\left(I+\frac{\tau^{2} A}{2}\right)^{-1} \\
& \left.\times\left(\frac{\widetilde{R}^{\left[\lambda_{m} / \tau\right]-1} R^{-1}\left(I-(i \tau / 2) A^{1 / 2}\right)+R^{\left[\lambda_{m} / \tau\right]-1} \widetilde{R}^{-1}\left(I+(i \tau / 2) A^{1 / 2}\right)}{2}\right)\right] \\
& +\left[\sum_{m=1}^{n} \alpha_{m}\left(I+\frac{\tau^{2} A}{2}\right)^{-1}\left(i A^{1 / 2}\right)^{-1} \frac{\widetilde{R}^{\left[\lambda_{m} / \tau\right]-1} R^{-1}-R^{\left[\lambda_{m} / \tau\right]-1} \widetilde{R}^{-1}}{2}\right. \\
& +\sum_{m=1}^{n} \alpha_{m}\left(\lambda_{m}-\left[\frac{\lambda_{m}}{\tau}\right] \tau\right)\left(I+\frac{\tau^{2} A}{2}\right)^{-1} \\
& \left.\times\left(\frac{\widetilde{R}^{\left[\lambda_{m} / \tau\right]-1} R^{-1}\left(I-(i \tau / 2) A^{1 / 2}\right)+R^{\left[\lambda_{m} / \tau\right]-1} \widetilde{R}^{-1}\left(I+(i \tau / 2) A^{1 / 2}\right)}{2}\right)\right] \\
& \times\left[\frac { \tau } { 2 } f _ { 0 } \left(\sum_{k=1}^{n} \beta_{k}\left(I+\frac{\tau^{2} A}{2}\right)^{-1}\right.\right. \\
& \times\left(\frac{\tilde{R}^{\left[\lambda_{k} / \tau\right]-1} R^{-1}\left(I-(i \tau / 2) A^{1 / 2}\right)}{2}+\frac{R^{\left[\lambda_{k} / \tau\right]-1} \tilde{R}^{-1}\left(I+(i \tau / 2) A^{1 / 2}\right)}{2}\right) \\
& -\sum_{k=1}^{n} \beta_{k}\left(\frac{\tau}{2}+\left(\lambda_{k}-\left[\frac{\lambda_{k}}{\tau}\right] \tau\right)\right) A \times\left(I+\frac{\tau^{2} A}{2}\right)^{-1}\left(i A^{1 / 2}\right)^{-1} \\
& \left.\times \frac{\tilde{R}^{\left[\lambda_{k} / \tau\right]-1} R^{-1}-R^{\left[\lambda_{k} / \tau\right]-1} \tilde{R}^{-1}}{2}\right)+\sum_{k=1}^{n} \beta_{k} \sum_{s=1}^{\left[\lambda_{k} / \tau\right]_{-2}} \frac{\tau}{2 i} \\
& \times A^{-1 / 2}\left(i A^{1 / 2}\right)\left(R^{\left[\lambda_{k} / \tau\right]-s}\left(I+\frac{i \tau}{2} A^{1 / 2}\right)+\tilde{R}^{\left[\lambda_{k} / \tau\right]-s}\left(I-\frac{i \tau}{2} A^{1 / 2}\right)\right) f_{s} \\
& +\sum_{k=1}^{n} \beta_{k} \tau R \tilde{R} f_{\left[\lambda_{k} / \tau\right]-1}+\sum_{k=1}^{n} \beta_{k}\left(\frac{\tau}{2}+\left(\lambda_{k}-\left[\frac{\lambda_{k}}{\tau}\right] \tau\right)\right) A \sum_{s=1}^{\left[\lambda_{k} / \tau\right]_{-1}} \frac{\tau}{2 i} A^{-1 / 2} \\
& \left.\left.\times\left(R^{\left[\lambda_{k} / \tau\right]-s}-\tilde{R}^{\left[\lambda_{k} / \tau\right]-s}\right) f_{s}+\sum_{k=1}^{n} \beta_{k}\left(\frac{\tau}{2}+\left(\lambda_{k}-\left[\frac{\lambda_{k}}{\tau}\right] \tau\right)\right) f_{\left[\lambda_{k} / \tau\right]-1}+\psi\right]\right\}, \\
& \omega=T_{\tau}\left\{\left[I-\sum_{m=1}^{n} \alpha_{m}\left(I+\frac{\tau^{2} A}{2}\right)^{-1}\right.\right. \\
& \times\left(\frac{R^{\left[\lambda_{m} / \tau\right]-1}\left(I-i \tau A^{1 / 2}\right)}{2}+\frac{\tilde{R}^{\left[\lambda_{m} / \tau\right]-1}\left(I+i \tau A^{1 / 2}\right)}{2}\right) \\
& -\sum_{m=1}^{n} \alpha_{m}\left(\lambda_{m}-\left[\frac{\lambda_{m}}{\tau}\right] \tau\right)\left(I+\frac{\tau^{2} A}{2}\right)^{-1}\left(i A^{1 / 2}\right)
\end{aligned}
$$




$$
\begin{aligned}
& \left.\times \frac{\widetilde{R}^{\left[\lambda_{m} / \tau\right]-1}\left(I-(i \tau / 2) A^{1 / 2}\right)\left(I+i \tau A^{1 / 2}\right)-R^{\left[\lambda_{m} / \tau\right]-1}\left(I+(i \tau / 2) A^{1 / 2}\right)\left(I-i \tau A^{1 / 2}\right)}{2}\right] \\
& \times\left[\frac { \tau } { 2 } f _ { 0 } \left(\sum_{k=1}^{n} \beta_{k}\left(I+\frac{\tau^{2} A}{2}\right)^{-1}\right.\right. \\
& \times\left(\frac{\widetilde{R}^{\left[\lambda_{k} / \tau\right]-1} R^{-1}\left(I-(i \tau / 2) A^{1 / 2}\right)}{2}+\frac{R^{\left[\lambda_{k} / \tau\right]-1} \widetilde{R}^{-1}\left(I+(i \tau / 2) A^{1 / 2}\right)}{2}\right) \\
& -\sum_{k=1}^{n} \beta_{k}\left(\frac{\tau}{2}+\left(\lambda_{k}-\left[\frac{\lambda_{k}}{\tau}\right] \tau\right)\right) A \times\left(I+\frac{\tau^{2} A}{2}\right)^{-1}\left(i A^{1 / 2}\right)^{-1} \\
& \left.\times \frac{\widetilde{R}^{\left[\lambda_{k} / \tau\right]-1} R^{-1}-R^{\left[\lambda_{k} / \tau\right]-1} \tilde{R}^{-1}}{2}\right)+\sum_{k=1}^{n} \beta_{k} \sum_{s=1}^{\left[\lambda_{k} / \tau\right]_{-2}} \frac{\tau}{2 i} \\
& \times A^{-1 / 2}\left(i A^{1 / 2}\right)\left(R^{\left[\lambda_{k} / \tau\right]-s}\left(I+\frac{i \tau}{2} A^{1 / 2}\right)+\tilde{R}^{\left[\lambda_{k} / \tau\right]-s}\left(I-\frac{i \tau}{2} A^{1 / 2}\right)\right) f_{s} \\
& +\sum_{k=1}^{n} \beta_{k} R \widetilde{R} f_{\left[\lambda_{k} / \tau\right]-1}+\sum_{k=1}^{n} \beta_{k}\left(\frac{\tau}{2}+\left(\lambda_{k}-\left[\frac{\lambda_{k}}{\tau}\right] \tau\right)\right) A \sum_{s=1}^{\left[\lambda_{k} / \tau\right]_{-1}} \frac{\tau}{2 i} \\
& \left.\times A^{-1 / 2}\left(R^{\left[\lambda_{k} / \tau\right]-s}-\tilde{R}^{\left[\lambda_{k} / \tau\right]-s}\right) f_{s}+\left(\frac{\tau}{2}+\left(\lambda_{k}-\left[\frac{\lambda_{k}}{\tau}\right] \tau\right)\right) f_{\left[\lambda_{k} / \tau\right]-1}+\psi\right] \\
& -\left[\sum_{k=1}^{n} \beta_{k} \frac{\tau}{2} A\left(I+\frac{\tau^{2} A}{2}\right)^{-1} \frac{R^{\left[\lambda_{k} / \tau\right]-1}\left(I-i \tau A^{1 / 2}\right)+\widetilde{R}^{\left[\lambda_{k} / \tau\right]-1}\left(I+i \tau A^{1 / 2}\right)}{2}\right. \\
& -\frac{1}{2} \sum_{k=1}^{n} \beta_{k}\left(I+\frac{\tau^{2} A}{2}\right)^{-1}\left(\tilde{R}^{\left[\lambda_{k} / \tau\right]-1}\left(I-\frac{i \tau}{2} A^{1 / 2}\right)\left(I+i \tau A^{1 / 2}\right)\right. \\
& \left.\left.-R^{\left[\lambda_{k} / \tau\right]-1}\left(I+\frac{i \tau}{2} A^{1 / 2}\right)\left(I-i \tau A^{1 / 2}\right)\right)\right] \\
& \times\left[\frac { \tau } { 2 } f _ { 0 } \left(\sum_{m=1}^{n} \alpha_{m}\left(I+\frac{\tau^{2} A}{2}\right)^{-1}\left(i A^{1 / 2}\right)^{-1} \frac{\widetilde{R}^{\left[\lambda_{m} / \tau\right]-1} R^{-1}-R^{\left[\lambda_{m} / \tau\right]-1} \widetilde{R}^{-1}}{2}\right.\right. \\
& +\sum_{m=1}^{n} \alpha_{m}\left(\lambda_{m}-\left[\frac{\lambda_{m}}{\tau}\right] \tau\right)\left(I+\frac{\tau^{2} A}{2}\right)^{-1} \\
& \left.\times\left(\frac{\widetilde{R}^{\left[\lambda_{m} / \tau\right]-1} R^{-1}\left(I-(i \tau / 2) A^{1 / 2}\right)+R^{\left[\lambda_{m} / \tau\right]-1} \widetilde{R}^{-1}\left(I+(i \tau / 2) A^{1 / 2}\right)}{2}\right)\right) \\
& -\left(\sum_{m=1}^{n} \alpha_{m} \sum_{s=1}^{\left[\lambda_{m} / \tau\right]_{-1}} \frac{\tau}{2 i} A^{-1 / 2}\left(R^{\left[\lambda_{m} / \tau\right]-s}-\widetilde{R}^{\left[\lambda_{m} / \tau\right]-s}\right) f_{s}+\sum_{m=1}^{n} \alpha_{m}\left(\lambda_{m}-\left[\frac{\lambda_{m}}{\tau}\right] \tau\right)\right. \\
& \times \sum_{s=1}^{\left[\lambda_{m} / \tau\right]_{-2}} \frac{\tau}{2 i} A^{-1 / 2}\left(i A^{1 / 2}\right)\left[R^{\left[\lambda_{m} / \tau\right]-S}\left(I+\frac{i \tau}{2} A^{1 / 2}\right)+\widetilde{R}^{\left[\lambda_{m} / \tau\right]-S}\left(I-\frac{i \tau}{2} A^{1 / 2}\right)\right] f_{S}
\end{aligned}
$$


Abstract and Applied Analysis

$$
\left.\left.\left.+\sum_{m=1}^{n} \alpha_{m} \tau\left(\lambda_{m}-\left[\frac{\lambda_{m}}{\tau}\right] \tau\right) R \tilde{R} f_{\left[\lambda_{m} / \tau\right]-1}-\varphi\right)\right]\right\} .
$$

Thus, formulas (2.9) and (2.10) give a solution of problem (2.1).

Theorem 2.3. Suppose that assumption (2.2) holds and $\varphi \in D(A), \psi \in D\left(A^{1 / 2}\right)$. Then, for the solution of difference scheme (2.1) the stability inequalities

$$
\begin{aligned}
& \max _{0 \leq k \leq N}\left\|u_{k}\right\|_{H} \leq M\left\{\sum_{k=0}^{N-1}\left\|A^{-1 / 2} f_{k}\right\|_{H} \tau+\left\|A^{-1 / 2} \psi\right\|_{H}+\|\varphi\|_{H}\right\}, \\
& \max _{0 \leq k \leq N}\left\|A^{1 / 2} u_{k}\right\|_{H} \leq M\left\{\sum_{k=0}^{N-1}\left\|f_{k}\right\|_{H} \tau+\left\|A^{1 / 2} \varphi\right\|_{H}+\|\varphi\|_{H}\right\}, \\
& \max _{1 \leq k \leq N-1}\left\|\tau^{-2}\left(u_{k+1}-2 u_{k}+u_{k-1}\right)\right\|_{H}+\max _{0 \leq k \leq N-1}\left\|A u_{k}+\frac{\tau^{2}}{4} A^{2} u_{k+1}\right\|_{H} \\
& \leq M\left\{\sum_{k=1}^{N-1}\left\|f_{k}-f_{k-1}\right\|_{H}+\left\|f_{0}\right\|_{H}+\left\|A^{1 / 2} \psi\right\|_{H}+\|A \varphi\|_{H}\right\}
\end{aligned}
$$

hold, where $M$ does not depend on $\tau, \varphi, \psi$, and $f_{k}, 0 \leq k \leq N-1$.

Proof. By [18], the following estimates

$$
\begin{gathered}
\max _{0 \leq k \leq N}\left\|u_{k}\right\|_{H} \leq M\left\{\sum_{k=0}^{N-1}\left\|A^{-1 / 2} f_{k}\right\|_{H} \tau+\left\|A^{-1 / 2} \omega\right\|_{H}+\|\mu\|_{H}\right\}, \\
\max _{0 \leq k \leq N}\left\|A^{1 / 2} u_{k}\right\|_{H} \leq M\left\{\sum_{k=0}^{N-1}\left\|f_{k}\right\|_{H} \tau+\left\|A^{1 / 2} \mu\right\|_{H}+\|\omega\|_{H}\right\}, \\
\max _{1 \leq k \leq N-1}\left\|\tau^{-2}\left(u_{k+1}-2 u_{k}+u_{k-1}\right)\right\|_{H}+\max _{0 \leq k \leq N-1}\left\|A u_{k}+\frac{\tau^{2}}{4} A^{2} u_{k+1}\right\|_{H} \\
\leq M\left\{\sum_{k=1}^{N-1}\left\|f_{k}-f_{k-1}\right\|_{H}+\left\|f_{0}\right\|_{H}+\left\|A^{1 / 2} \omega\right\|_{H}+\|A \mu\|_{H}\right\}
\end{gathered}
$$

hold for the solution of (2.8). Using formulas of $\mu, \omega$, and (2.3) and (2.6) the following estimates obtained

$$
\begin{gathered}
\|\mu\|_{H} \leq M\left\{\sum_{s=0}^{N-1}\left\|A^{-1 / 2} f_{s}\right\|_{H} \tau+\left\|A^{-1 / 2} \psi\right\|_{H}+\|\varphi\|_{H}\right\}, \\
\left\|A^{-1 / 2} \omega\right\|_{H} \leq M\left\{\sum_{s=0}^{N-1}\left\|A^{-1 / 2} f_{s}\right\|_{H} \tau+\left\|A^{-1 / 2} \psi\right\|_{H}+\|\varphi\|_{H}\right\} .
\end{gathered}
$$


Estimate (2.11) follows from (2.14), (2.17), and (2.18). In a similar manner, we obtain

$$
\max _{0 \leq k \leq N}\left\|A^{1 / 2} u_{k}\right\|_{H} \leq M\left\{\sum_{k=0}^{N-1}\left\|f_{k}\right\|_{H} \tau+\left\|A^{1 / 2} \varphi\right\|_{H}+\|\psi\|_{H}\right\} .
$$

Now, we obtain the estimates for $\|A \mu\|_{H}$ and $\left\|A^{1 / 2} \omega\right\|_{H}$. First, applying $A$ to the formula of $\mu$ and using Abel's formula, we can write

$$
\begin{aligned}
& A \mu=T_{\tau}\left\{\left[\frac { \tau } { 2 } f _ { 0 } \left(\sum_{m=1}^{n} \alpha_{m}\left(I+\frac{\tau^{2} A}{2}\right)^{-1} A^{1 / 2} \frac{\widetilde{R}^{\left[\lambda_{m} / \tau\right]-1} R^{-1}-R^{\left[\lambda_{m} / \tau\right]-1} \widetilde{R}^{-1}}{2 i}\right.\right.\right. \\
& +\sum_{m=1}^{n} \alpha_{m}\left(\lambda_{m}-\left[\frac{\lambda_{m}}{\tau}\right] \tau\right) A\left(I+\frac{\tau^{2} A}{2}\right)^{-1} \\
& \left.\times \frac{\widetilde{R}^{\left[\lambda_{m} / \tau\right]-1} R^{-1}\left(I-(i \tau / 2) A^{1 / 2}\right)+R^{\left[\lambda_{m} / \tau\right]-1} \widetilde{R}^{-1}\left(I+(i \tau / 2) A^{1 / 2}\right)}{2}\right) \\
& -\left(\frac{1}{2} \sum_{m=1}^{n} \alpha_{m} \sum_{s=2}^{\left[\lambda_{m} / \tau\right]}\left(R^{\left[\lambda_{m} / \tau\right]-\mathrm{s}}\left(I+\frac{i \tau}{2} A^{1 / 2}\right)^{-1}+\widetilde{R}^{\left[\lambda_{m} / \tau\right]-\mathrm{s}}\left(I-\frac{i \tau}{2} A^{1 / 2}\right)^{-1}\right)\right. \\
& \times\left(f_{s}-f_{s-1}\right)+\sum_{m=1}^{n} \alpha_{m} \frac{1}{2}\left(R^{\left[\lambda_{m} / \tau\right]-1}\left(I+\frac{i \tau}{2} A^{1 / 2}\right)^{-1}+\tilde{R}^{\left[\lambda_{m} / \tau\right]-1}\left(I-\frac{i \tau}{2} A^{1 / 2}\right)^{-1}\right) f_{1} \\
& -\sum_{m=1}^{n} \alpha_{m}\left(I+\frac{\tau^{2} A}{4}\right)^{-1} f_{\left[\lambda_{m} / \tau\right]-1}+\sum_{m=1}^{n} \alpha_{m}\left(\lambda_{m}-\left[\frac{\lambda_{m}}{\tau}\right] \tau\right) \\
& \times\left[\sum_{s=2}^{\left[\lambda_{m} / \tau\right]_{-2}} \frac{1}{2 i} A^{1 / 2} \times\left(R^{\left[\lambda_{m} / \tau\right]-s}+\tilde{R}^{\left[\lambda_{m} / \tau\right]-s}\right)\left(f_{s}-f_{s-1}\right)\right. \\
& \left.+\frac{1}{2 i} A^{1 / 2}\left(R^{\left[\lambda_{m} / \tau\right]-1}+\widetilde{R}^{\left[\lambda_{m} / \tau\right]-1}\right) f_{1}+i A^{1 / 2} f_{\left[\lambda_{m} / \tau\right]-2}\right] \\
& \left.\left.+\sum_{m=1}^{n} \alpha_{m} \tau\left(\lambda_{m}-\left[\frac{\lambda_{m}}{\tau}\right] \tau\right) A R \widetilde{R} f_{\left[\lambda_{m} / \tau\right]-1}-A \varphi\right)\right] \\
& \times\left[I+\sum_{k=1}^{n} \beta_{k}\left(\frac{\tau}{2}+\left(\lambda_{k}-\left[\frac{\lambda_{k}}{\tau}\right] \tau\right)\right) A\left(I+\frac{\tau^{2} A}{2}\right)^{-1}\left(i A^{1 / 2}\right)^{-1}\right. \\
& \times\left(\frac{\tilde{R}^{\left[\lambda_{k} / \tau\right]-1} R^{-1}-R^{\left[\lambda_{k} / \tau\right]-1} \tilde{R}^{-1}}{2}\right)-\sum_{m=1}^{n} \alpha_{m}\left(\lambda_{m}-\left[\frac{\lambda_{m}}{\tau}\right] \tau\right)\left(I+\frac{\tau^{2} A}{2}\right)^{-1} \\
& \left.\times \frac{\widetilde{R}^{\left[\lambda_{m} / \tau\right]-1} R^{-1}\left(I-(i \tau / 2) A^{1 / 2}\right)+R^{\left[\lambda_{m} / \tau\right]-1} \widetilde{R}^{-1}\left(I+(i \tau / 2) A^{1 / 2}\right)}{2}\right]
\end{aligned}
$$




$$
\begin{gathered}
+\left[\sum_{m=1}^{n} \alpha_{m}\left(I+\frac{\tau^{2} A}{2}\right)^{-1} \frac{\tilde{R}^{\left[\lambda_{m} / \tau\right]-1} R^{-1}-R^{\left[\lambda_{m} / \tau\right]-1} \tilde{R}^{-1}}{2 i}+\sum_{m=1}^{n} \alpha_{m}\left(\lambda_{m}-\left[\frac{\lambda_{m}}{\tau}\right] \tau\right)\right. \\
\times A^{1 / 2}\left(I+\frac{\tau^{2} A}{2}\right)^{-1} \\
\left.\times \frac{\tilde{R}^{\left[\lambda_{m} / \tau\right]-1} R^{-1}\left(I-(i \tau / 2) A^{1 / 2}\right)+R^{\left[\lambda_{m} / \tau\right]-1} \widetilde{R}^{-1}\left(I+(i \tau / 2) A^{1 / 2}\right)}{2}\right] \\
\times\left[\frac { \tau } { 2 } f _ { 0 } \left(\sum_{k=1}^{n} \beta_{k} A^{1 / 2}\left(I+\frac{\tau^{2} A}{2}\right)^{-1}\right.\right. \\
\times\left(\frac{\tilde{R}^{\left[\lambda_{k} / \tau\right]-1} R^{-1}\left(I-(i \tau / 2) A^{1 / 2}\right)}{2}+\frac{R^{\left[\lambda_{k} / \tau\right]-1} \widetilde{R}^{-1}\left(I+(i \tau / 2) A^{1 / 2}\right)}{2}\right) \\
+\sum_{k=1}^{n} \beta_{k} i\left(\frac{\tau}{2}+\left(\lambda_{k}-\left[\frac{\lambda_{k}}{\tau}\right] \tau\right)\right) A \times\left(I+\frac{\tau^{2} A}{2}\right)^{-1} \\
\left.\times \frac{\tilde{R}^{\left[\lambda_{k} / \tau\right]-1} R^{-1}-R^{\left[\lambda_{k} / \tau\right]-1} \widetilde{R}^{-1}}{2}\right)
\end{gathered}
$$

$-\sum_{k=1}^{n} \beta_{k} \sum_{s=2}^{\left[\lambda_{k} / \tau\right]_{-2}} \frac{1}{2 i}\left(R^{\left[\lambda_{k} / \tau\right]-s}+\tilde{R}^{\left[\lambda_{k} / \tau\right]-s}\right)\left(f_{s}-f_{s-1}\right)$

$-\sum_{k=1}^{n} \beta_{k} \frac{1}{2 i}\left(R^{\left[\lambda_{k} / \tau\right]-1}+\tilde{R}^{\left[\lambda_{k} / \tau\right]-1}\right) f_{1}-\sum_{k=1}^{n} \beta_{k} i f_{\left[\lambda_{m} / \tau\right]-2}$

$+\sum_{k=1}^{n} \beta_{k} A^{1 / 2} \tau R \tilde{R} f_{\left[\lambda_{k} / \tau\right]-1}+\sum_{k=1}^{n} \beta_{k}\left(\frac{\tau}{2}+\left(\lambda_{k}-\left[\frac{\lambda_{k}}{\tau}\right] \tau\right)\right)$

$\times\left[\frac{1}{2} A^{-1 / 2} \sum_{s=2}^{\left[\lambda_{k} / \tau\right]-1}\left(R^{\left[\lambda_{k} / \tau\right]-s}\left(I+\frac{i \tau}{2} A^{1 / 2}\right)^{-1}+\widetilde{R}^{\left[\lambda_{k} / \tau\right]-s}\left(I-\frac{i \tau}{2} A^{1 / 2}\right)^{-1}\right)\right.$

$\times\left(f_{s}-f_{s-1}\right)+\frac{1}{2} A^{-1 / 2}\left(R^{\left[\lambda_{k} / \tau\right]-1}\left(I+\frac{i \tau}{2} A^{1 / 2}\right)^{-1}+\widetilde{R}^{\left[\lambda_{k} / \tau\right]-1}\left(I-\frac{i \tau}{2} A^{1 / 2}\right)^{-1}\right) f_{1}$

$$
\left.-A^{-1 / 2}\left(I+\frac{\tau^{2} A}{4}\right)^{-1} f_{\left[\lambda_{k} / \tau\right]-1}\right]
$$

$\left.\left.+\sum_{k=1}^{n} \beta_{k}\left(\frac{\tau}{2}+\left(\lambda_{k}-\left[\frac{\lambda_{k}}{\tau}\right] \tau\right)\right) A^{1 / 2} f_{\left[\lambda_{k} / \tau\right]-1}+A^{1 / 2} \psi\right]\right\}$. 
Second, applying $A^{1 / 2}$ to the formula of $\omega$ and using Abel's formula, we can write

$$
\begin{aligned}
& A^{1 / 2} \omega=T_{\tau}\left\{\left[I-\sum_{m=1}^{n} \alpha_{m}\left(I+\frac{\tau^{2} A}{2}\right)^{-1}\right.\right. \\
& \times\left(\frac{R^{\left[\lambda_{m} / \tau\right]-1}\left(I-i \tau A^{1 / 2}\right)}{2}+\frac{\widetilde{R}^{\left[\lambda_{m} / \tau\right]-1}\left(I+i \tau A^{1 / 2}\right)}{2}\right) \\
& -\sum_{m=1}^{n} \alpha_{m}\left(\lambda_{m}-\left[\frac{\lambda_{m}}{\tau}\right] \tau\right)\left(I+\frac{\tau^{2} A}{2}\right)^{-1}\left(i A^{1 / 2}\right) \\
& \left.\times \frac{\widetilde{R}^{\left[\lambda_{m} / \tau\right]-1}\left(I-(i \tau / 2) A^{1 / 2}\right)\left(I+i \tau A^{1 / 2}\right)-R^{\left[\lambda_{m} / \tau\right]-1}\left(I+(i \tau / 2) A^{1 / 2}\right)\left(I-i \tau A^{1 / 2}\right)}{2}\right] \\
& \times\left[\frac { 1 } { 2 } f _ { 0 } \left(\sum_{k=1}^{n} \beta_{k} A^{1 / 2} \tau\left(I+\frac{\tau^{2} A}{2}\right)^{-1}\right.\right. \\
& \times\left(\frac{\widetilde{R}^{\left[\lambda_{k} / \tau\right]-1} R^{-1}\left(I-(i \tau / 2) A^{1 / 2}\right)}{2}+\frac{R^{\left[\lambda_{k} / \tau\right]-1} \widetilde{R}^{-1}\left(I+(i \tau / 2) A^{1 / 2}\right)}{2}\right) \\
& +\sum_{k=1}^{n} \beta_{k}\left(\frac{\tau}{2}+\left(\lambda_{k}-\left[\frac{\lambda_{k}}{\tau}\right] \tau\right)\right)\left(I+\frac{\tau^{2} A}{2}\right)^{-1} \\
& \left.\times A^{1 / 2} i \frac{\widetilde{R}^{\left[\lambda_{k} / \tau\right]-1} R^{-1}-R^{\left[\lambda_{k} / \tau\right]-1} \tilde{R}^{-1}}{2}\right) \\
& -\sum_{k=1}^{n} \beta_{k} \sum_{s=2}^{\left[\lambda_{k} / \tau\right]-2} \frac{1}{2 i}\left(R^{\left[\lambda_{k} / \tau\right]-\mathrm{s}}+\widetilde{R}^{\left[\lambda_{k} / \tau\right]-\mathrm{s}}\right) \\
& \times\left(f_{s}-f_{s-1}\right)-\sum_{k=1}^{n} \beta_{k} \frac{1}{2 i}\left(R^{\left[\lambda_{k} / \tau\right]-1}+\widetilde{R}^{\left[\lambda_{k} / \tau\right]-1}\right) f_{1}-\sum_{k=1}^{n} \beta_{k} i f_{\left[\lambda_{k} / \tau\right]-2} \\
& +\sum_{k=1}^{n} \beta_{k} A^{1 / 2} \tau R \tilde{R} f_{\left[\lambda_{k} / \tau\right]-1}+\sum_{k=1}^{n} \beta_{k}\left(\frac{\tau}{2}+\left(\lambda_{k}-\left[\frac{\lambda_{k}}{\tau}\right] \tau\right)\right) A^{-1 / 2} \\
& \times\left[\frac{1}{2} \sum_{s=2}^{\left[\lambda_{k} / \tau\right]-1}\left(R^{\left[\lambda_{k} / \tau\right]-s}\left(I+\frac{i \tau}{2} A^{1 / 2}\right)^{-1}+\widetilde{R}^{\left[\lambda_{k} / \tau\right]-s}\left(I-\frac{i \tau}{2} A^{1 / 2}\right)^{-1}\right)\right. \\
& \times\left(f_{s}-f_{s-1}\right)+\frac{1}{2}\left(R^{\left[\lambda_{k} / \tau\right]-1}\left(I+\frac{i \tau}{2} A^{1 / 2}\right)^{-1}+\widetilde{R}^{\left[\lambda_{k} / \tau\right]-1}\left(I-\frac{i \tau}{2} A^{1 / 2}\right)^{-1}\right) f_{1} \\
& \left.\left.-\left(I+\frac{\tau^{2} A}{4}\right)^{-1} f_{\left[\lambda_{k} / \tau\right]-1}\right]+A^{1 / 2} \psi\right]
\end{aligned}
$$


Abstract and Applied Analysis

$$
\begin{aligned}
& -\left[\sum_{k=1}^{n} \beta_{k} A^{1 / 2} \frac{\tau}{2}\left(I+\frac{\tau^{2} A}{2}\right)^{-1} \times\left(\frac{R^{\left[\lambda_{k} / \tau\right]-1}\left(I-i \tau A^{1 / 2}\right)+\tilde{R}^{\left[\lambda_{k} / \tau\right]-1}\left(I+i \tau A^{1 / 2}\right)}{2}\right)\right. \\
& -\sum_{k=1}^{n} \beta_{k} A^{-1 / 2}\left(I+\frac{\tau^{2} A}{2}\right)^{-1} \\
& \times\left(\frac{\widetilde{R}^{\left[\lambda_{k} / \tau\right]-1}\left(I-(i \tau / 2) A^{1 / 2}\right)\left(I+i \tau A^{1 / 2}\right)}{2}\right. \\
& \left.\left.-\frac{R^{\left[\lambda_{k} / \tau\right]-1}\left(I+(i \tau / 2) A^{1 / 2}\right)\left(I-i \tau A^{1 / 2}\right)}{2}\right)\right] \\
& \times\left[\frac { 1 } { 2 } f _ { 0 } \left(\sum_{m=1}^{n} \alpha_{m}\left(I+\frac{\tau^{2} A}{2}\right)^{-1} \times \tau A^{1 / 2} \frac{\tilde{R}^{\left[\lambda_{m} / \tau\right]-1} R^{-1}-R^{\left[\lambda_{m} / \tau\right]-1} \tilde{R}^{-1}}{2 i}\right.\right. \\
& +\sum_{m=1}^{n} \alpha_{m}\left(\lambda_{m}-\left[\frac{\lambda_{m}}{\tau}\right] \tau\right) A\left(I+\frac{\tau^{2} A}{2}\right)^{-1} \\
& \left.\times\left(\frac{\widetilde{R}^{\left[\lambda_{m} / \tau\right]-1} R^{-1}\left(I-(i \tau / 2) A^{1 / 2}\right)+R^{\left[\lambda_{m} / \tau\right]-1} \tilde{R}^{-1}\left(I+(i \tau / 2) A^{1 / 2}\right)}{2}\right)\right) \\
& -\left(\frac{1}{2} \sum_{m=1}^{n} \alpha_{m} A^{-1 / 2}\right. \\
& \times \sum_{s=2}^{\left[\lambda_{m} / \tau\right]-1}\left(R^{\left[\lambda_{m} / \tau\right]-s}\left(I+\frac{i \tau}{2} A^{1 / 2}\right)^{-1}+\widetilde{R}^{\left[\lambda_{m} / \tau\right]-s}\left(I-\frac{i \tau}{2} A^{1 / 2}\right)^{-1}\right) \\
& \times\left(f_{s}-f_{s-1}\right)+\frac{1}{2} \sum_{m=1}^{n} \alpha_{m} A^{-1 / 2} \\
& \times\left(R^{\left[\lambda_{m} / \tau\right]-1}\left(I+\frac{i \tau}{2} A^{1 / 2}\right)^{-1}+\widetilde{R}^{\left[\lambda_{m} / \tau\right]-1}\left(I-\frac{i \tau}{2} A^{1 / 2}\right)^{-1}\right) f_{1} \\
& -\sum_{m=1}^{n} \alpha_{m} A^{-1 / 2}\left(I+\frac{\tau^{2} A}{4}\right)^{-1} f_{\left[\lambda_{m} / \tau\right]-1}-\sum_{m=1}^{n} \alpha_{m}\left(\lambda_{m}-\left[\frac{\lambda_{m}}{\tau}\right] \tau\right) \\
& \times\left[\sum_{s=2}^{\left[\lambda_{m} / \tau\right]-2} \frac{1}{2 i} A^{1 / 2} \times\left(R^{\left[\lambda_{m} / \tau\right]-s}+\widetilde{R}^{\left[\lambda_{m} / \tau\right]-s}\right)\left(f_{s}-f_{s-1}\right)\right. \\
& \left.+\frac{1}{2 i} A^{1 / 2}\left(R^{\left[\lambda_{m} / \tau\right]-1}+\tilde{R}^{\left[\lambda_{m} / \tau\right]-1}\right) f_{1}+i A^{1 / 2} f_{\left[\lambda_{m} / \tau\right]-2}\right] \\
& \left.\left.\left.+\sum_{m=1}^{n} \alpha_{m} \tau\left(\lambda_{m}-\left[\frac{\lambda_{m}}{\tau}\right] \tau\right) A R \widetilde{R} f_{\left[\lambda_{m} / \tau\right]-1}-A \varphi\right)\right]\right\} .
\end{aligned}
$$

The following estimates 


$$
\begin{gathered}
\|A \mu\|_{H} \leq M\left\{\sum_{s=1}^{N-1}\left\|f_{s}-f_{s-1}\right\|_{H}+\left\|f_{0}\right\|_{H}+\left\|A^{1 / 2} \psi\right\|_{H}+\|A \varphi\|_{H}\right\}, \\
\left\|A^{1 / 2} \omega\right\|_{H} \leq M\left\{\sum_{s=1}^{N-1}\left\|f_{s}-f_{s-1}\right\|_{H}+\left\|f_{0}\right\|_{H}+\left\|A^{1 / 2} \psi\right\|_{H}+\|A \varphi\|_{H}\right\}
\end{gathered}
$$

are obtained by using formulas (2.20), (2.21), (2.3), and (2.6).

Estimate (2.13) follows from (2.16), and (2.22). Theorem 2.3 is proved.

Now, let us consider the applications of Theorem 2.3. First, the nonlocal the mixed boundary value problem for hyperbolic equation

$$
\begin{aligned}
& u_{t t}-\left(a(x) u_{x}\right)_{x}+\delta u=f(t, x), \quad 0<t<1,0<x<1, \\
& u(0, x)=\sum_{m=1}^{n} \alpha_{m} u\left(\lambda_{m}, x\right)+\varphi(x), \quad 0 \leq x \leq 1, \\
& u_{t}(0, x)=\sum_{k=1}^{n} \beta_{k} u_{t}\left(\lambda_{k}, x\right)+\psi(x), \quad 0 \leq x \leq 1, \\
& u(t, 0)=u(t, 1), \quad u_{x}(t, 0)=u_{x}(t, 1), \quad 0 \leq t \leq 1,
\end{aligned}
$$

under assumption (2.2), is considered. Here $a_{r}(x),(x \in(0,1)), \varphi(x), \psi(x)(x \in[0,1])$ and $f(t, x)(t \in(0,1), x \in(0,1))$ are given smooth functions and $a_{r}(x) \geq a>0, \delta>0$. The discretization of problem (2.23) is carried out in two steps.

In the first step, the grid space is defined as follows:

$$
[0,1]_{h}=\left\{x: x_{r}=r h, 0 \leq r \leq K, K h=1\right\} .
$$

We introduce the Hilbert space $L_{2 h}=L_{2}\left([0,1]_{h}\right), W_{2 h}^{1}=W_{2 h}^{1}\left([0,1]_{h}\right)$ and $W_{2 h}^{2}=W_{2 h}^{2}\left([0,1]_{h}\right)$ of the grid functions $\varphi^{h}(x)=\left\{\varphi_{r}\right\}_{1}^{K-1}$ defined on $[0,1]_{h}$, equipped with the norms

$$
\begin{gathered}
\left\|\varphi^{h}\right\|_{L_{2 h}}=\left(\sum_{r=1}^{K-1}\left|\varphi^{h}(x)\right|^{2} h\right)^{1 / 2}, \\
\left\|\varphi^{h}\right\|_{W_{2 h}^{1}}=\left\|\varphi^{h}\right\|_{L_{2 h}}+\left(\sum_{r=1}^{K-1}\left|\left(\varphi^{h}\right)_{\bar{x}, j}\right|^{2} h\right)^{1 / 2}, \\
\left\|\varphi^{h}\right\|_{W_{2 h}^{2}}=\left\|\varphi^{h}\right\|_{L_{2 h}}+\left(\sum_{r=1}^{K-1}\left|\left(\varphi^{h}\right)_{\bar{x}, j}\right|^{2} h\right)^{1 / 2}+\left(\sum_{r=1}^{K-1}\left|\left(\varphi^{h}\right)_{x \bar{x}, j}\right|^{2} h\right)^{1 / 2},
\end{gathered}
$$

respectively. To the differential operator $A$ generated by problem (2.23), we assign the difference operator $A_{h}^{x}$ by the formula

$$
A_{h}^{x} \varphi^{h}(x)=\left\{-\left(a(x) \varphi_{\bar{x}}\right)_{x, r}+\delta \varphi_{r}\right\}_{1}^{K-1},
$$

acting in the space of grid functions $\varphi^{h}(x)=\left\{\varphi_{r}\right\}_{0}^{K}$ satisfying the conditions $\varphi_{0}=\varphi_{K}, \varphi_{1}-\varphi_{0}=$ $\varphi_{K}-\varphi_{K-1}$. With the help of $A_{h^{\prime}}^{x}$ we arrive at the nonlocal boundary value problem 
Abstract and Applied Analysis

$$
\begin{array}{ll}
\frac{d^{2} v^{h}(t, x)}{d t^{2}}+A_{h}^{x} v^{h}(t, x)=f^{h}(t, x), & 0 \leq t \leq 1, x \in[0,1]_{h}, \\
v^{h}(0, x)=\sum_{j=1}^{n} \alpha_{j} v^{h}\left(\lambda_{j}, x\right)+\varphi^{h}(x), & x \in[0,1]_{h^{\prime}} \\
v_{t}^{h}(0, x)=\sum_{j=1}^{n} \beta_{j} v_{t}^{h}\left(\lambda_{j}, x\right)+\psi^{h}(x), & x \in[0,1]_{h^{\prime}}
\end{array}
$$

for an infinite system of ordinary differential equations.

In the second step, we replace problem (2.27) by difference scheme (2.28)

$$
\begin{gathered}
\frac{u_{k+1}^{h}(x)-2 u_{k}^{h}(x)+u_{k-1}^{h}(x)}{\tau^{2}}+A_{h}^{x} u_{k}^{h}(x)+\frac{\tau^{2}}{4}\left(A_{h}^{x}\right)^{2} u_{k+1}^{h}(x)=f_{k}^{h}(x), \quad x \in[0,1]_{h^{\prime}}, \\
f_{k}^{h}(x)=f^{h}\left(t_{k}, x\right), \quad t_{k}=k \tau, 1 \leq k \leq N-1, N \tau=1, \\
u_{0}^{h}(x)=\sum_{j=1}^{h} \alpha_{j}\left\{u_{\left[\lambda_{j} / \tau\right]}^{h}(x)+\tau^{-1}\left(u_{\left[\lambda_{j} / \tau\right]}^{h}(x)-u_{\left[\lambda_{j} / \tau\right]-1}^{h}(x)\right)\left(\lambda_{j}-\left[\frac{\lambda_{j}}{\tau}\right] \tau\right)\right\}+\varphi^{h}(x), \quad x \in[0,1]_{h}, \\
\left(I+\frac{\tau^{2} A_{h}^{x}}{2}\right) \frac{u_{1}^{h}(x)-u_{0}^{h}(x)}{\tau}-\frac{\tau}{2}\left(f_{0}^{h}(x)-A_{h}^{x} u_{0}^{h}(x)\right) \\
=\sum_{j=1}^{n} \beta_{j}\left\{\frac{u_{\left[\lambda_{j} / \tau\right]}^{h}(x)-u_{\left[\lambda_{j} / \tau\right]-1}^{h}(x)}{\tau}+\left(\frac{\tau}{2}+\left(\lambda_{j}-\left[\frac{\lambda_{j}}{\tau}\right] \tau\right)\right) \times\left(f_{\left[\lambda_{j} / \tau\right]}^{h}(x)-A_{h}^{x} u_{\left[\lambda_{j} / \tau\right]}^{h}(x)\right)\right\} \\
+\psi^{h}(x), \quad x \in[0,1]_{h} .
\end{gathered}
$$

Theorem 2.4. Let $\tau$ and $h$ be sufficiently small positive numbers. Suppose that assumption (2.2) holds. Then, the solution of difference scheme (2.28) satisfies the following stability estimates:

$$
\begin{aligned}
& \max _{0 \leq k \leq N}\left\|u_{k}^{h}\right\|_{L_{2 h}}+\max _{0 \leq k \leq N}\left\|\left(u_{k}^{h}\right)_{x}\right\|_{L_{2 h}} \\
& \quad \leq M_{1}\left[\max _{0 \leq k \leq N-1}\left\|f_{k}^{h}\right\|_{L_{2 h}}+\left\|\psi^{h}\right\|_{L_{2 h}}+\left\|\varphi_{\bar{x}}^{h}\right\|_{L_{2 h}}\right], \\
& \max _{1 \leq k \leq N-1}\left\|\tau^{-2}\left(u_{k+1}^{h}-2 u_{k}^{h}+u_{k-1}^{h}\right)\right\|_{L_{2 h}}+\max _{0 \leq k \leq N}\left\|\left(u_{k}^{h}\right)_{\bar{x} x}\right\|_{L_{2 h}} \\
& \quad \leq M_{1}\left[\left\|f_{0}^{h}\right\|_{L_{2 h}}+\max _{1 \leq k \leq N-1}\left\|\tau^{-1}\left(f_{k}^{h}-f_{k-1}^{h}\right)\right\|_{L_{2 h}}+\left\|\psi_{\bar{x}}^{h}\right\|_{L_{2 h}}+\left\|\left(\varphi_{\bar{x}}^{h}\right)_{x}\right\|_{L_{2 h}}\right] .
\end{aligned}
$$

Here, $M_{1}$ does not depend on $\tau, h, \varphi^{h}(x), \psi^{h}(x)$ and $f_{k^{\prime}}^{h} 0 \leq k<N$.

The proof of Theorem 2.4 is based on abstract Theorem 2.3 and symmetry properties of the operator $A_{h}^{x}$ defined by (2.26). 
Second, for the $m$-dimensional hyperbolic equation under assumption (2.2) is considered. Let $\Omega$ be the unit open cube in the $m$-dimensional Euclidean space $\mathbb{R}^{m}\left\{x=\left(x_{1}, \ldots, x_{m}\right)\right.$ : $\left.0<x_{j}<1,1 \leq j \leq m\right\}$ with boundary $S, \bar{\Omega}=\Omega \cup S$. In $[0,1] \times \Omega$, the mixed boundary value problem for the multidimensional hyperbolic equation

$$
\begin{aligned}
& \frac{\partial^{2} u(t, x)}{\partial t^{2}}-\sum_{r=1}^{m}\left(a_{r}(x) u_{x_{r}}\right)_{x_{r}}=f(t, x), \\
& x=\left(x_{1}, \ldots, x_{m}\right) \in \Omega, \quad 0<t<1, \\
& u(0, x)=\sum_{j=1}^{n} \alpha_{j} u\left(\lambda_{j}, x\right)+\varphi(x), \quad x \in \overline{\Omega ;} \\
& u_{t}(0, x)=\sum_{k=1}^{n} \beta_{k} u_{t}\left(\lambda_{k}, x\right)+\psi(x), \quad x \in \overline{\Omega ;} \\
& u(t, x)=0, \quad x \in S
\end{aligned}
$$

is considered.

Here, $a_{r}(x),(x \in \Omega), \varphi(x), \psi(x)(x \in \bar{\Omega})$ and $f(t, x)(t \in(0,1), x \in \Omega)$ are given smooth functions and $a_{r}(x) \geq a>0$. The discretization of problem (2.30) is carried out in two steps. In the first step, let us define the grid sets

$$
\begin{gathered}
\widetilde{\Omega}_{h}=\left\{x=x_{r}=\left(h_{1} r_{1}, \ldots, h_{m} r_{m}\right), r=\left(r_{1}, \ldots, r_{m}\right), 0 \leq r_{j} \leq N_{j}, h_{j} N_{j}=1, j=1, \ldots, m\right\}, \\
\Omega_{h}=\widetilde{\Omega}_{h} \cap \Omega, \quad S_{h}=\widetilde{\Omega}_{h} \cap S .
\end{gathered}
$$

We introduce the Banach space $L_{2 h}=L_{2}\left(\tilde{\Omega}_{h}\right), W_{2 h}^{1}=W_{2 h}^{1}\left(\tilde{\Omega}_{h}\right)$ and $W_{2 h}^{2}=W_{2 h}^{2}\left(\tilde{\Omega}_{h}\right)$ of the grid functions $\varphi^{h}(x)=\left\{\varphi\left(h_{1} r_{1}, \ldots, h_{m} r_{m}\right)\right\}$ defined on $\widetilde{\Omega}_{h}$, equipped with the norms

$$
\begin{gathered}
\left\|\varphi^{h}\right\|_{L_{2}\left(\tilde{\Omega}_{h}\right)}=\left(\sum_{x \in \overline{\Omega_{h}}}\left|\varphi^{h}(x)\right|^{2} h_{1} \cdots h_{m}\right)^{1 / 2}, \\
\left\|\varphi^{h}\right\|_{W_{2 h}^{1}}=\left\|\varphi^{h}\right\|_{L_{2 h}}+\left(\sum_{x \in \overline{\Omega_{h}}} \sum_{r=1}^{m}\left|\left(\varphi^{h}\right)_{\overline{x_{r}, j_{r}}}\right|^{2} h_{1} \cdots h_{m}\right)^{1 / 2}, \\
\left\|\varphi^{h}\right\|_{W_{2 h}^{2}}=\left\|\varphi^{h}\right\|_{L_{2 h}}+\left(\sum_{x \in \overline{\Omega_{h}}} \sum_{r=1}^{m}\left|\left(\varphi^{h}\right)_{\overline{x_{r}}}\right|^{2} h_{1} \cdots h_{m}\right)^{1 / 2} \\
+\left(\sum_{x \in \overline{\Omega_{h}}} \sum_{r=1}^{m}\left|\left(\varphi^{h}\right)_{x_{r} \overline{x_{r}, j_{r}}}\right|^{2} h_{1} \cdots h_{m}\right)^{1 / 2},
\end{gathered}
$$


respectively. To the differential operator $A$ generated by problem (2.27), we assign the difference operator $A_{h}^{x}$ by the formula

$$
A_{h}^{x} u^{h}=-\sum_{r=1}^{m}\left(a_{r}(x) u_{\overline{x_{r}}}^{h}\right)_{x_{r}, j_{r}}
$$

acting in the space of grid functions $u^{h}(x)$, satisfying the conditions $u^{h}(x)=0$ for all $x \in S_{h}$. Note that $A_{h}^{x}$ is a self-adjoint positive definite operator in $L_{2}\left(\widetilde{\Omega}_{h}\right)$. With the help of $A_{h}^{x}$ we arrive at the nonlocal boundary value problem

$$
\begin{aligned}
& \frac{d^{2} v^{h}(t, x)}{d t^{2}}+A_{h}^{x} v^{h}(t, x)=f^{h}(t, x), \quad 0<t<1, x \in \Omega_{h}, \\
& v^{h}(0, x)=\sum_{l=1}^{n} \alpha_{l} v^{h}\left(\lambda_{l}, x\right)+\varphi^{h}(x), \quad x \in \widetilde{\Omega}_{h}, \\
& \frac{d v^{h}(0, x)}{d t}=\sum_{l=1}^{n} \beta_{l} v_{t}^{h}\left(\lambda_{l}, x\right)+\psi^{h}(x), \quad x \in \tilde{\Omega},
\end{aligned}
$$

for an infinite system of ordinary differential equations.

In the second step, we replace problem (2.34) by difference scheme (2.35)

$$
\begin{gathered}
\frac{u_{k+1}^{h}(x)-2 u_{k}^{h}(x)+u_{k-1}^{h}(x)}{\tau^{2}}+A_{h}^{x} u_{k}^{h}(x)+\frac{\tau^{2}}{4}\left(A_{h}^{x}\right)^{2} u_{k+1}^{h}(x)=f_{k}^{h}(x), \quad x \in \Omega_{h,}, \\
f_{k}^{h}(x)=f^{h}\left(t_{k}, x\right), \quad t_{k}=k \tau, 1 \leq k \leq N-1, N \tau=1, \\
u_{0}^{h}(x)=\sum_{l=1}^{n} \alpha_{l}\left\{u_{\left[\lambda_{l} / \tau\right]}^{h}(x)+\tau^{-1}\left(u_{\left[\lambda_{l} / \tau\right]}^{h}(x)-u_{\left[\lambda_{l} / \tau\right]-1}^{h}(x)\right)\left(\lambda_{l}-\left[\lambda_{l} / \tau\right] \tau\right)\right\}+\varphi^{h}(x), \quad x \in \widetilde{\Omega}_{h}, \\
\left(I+\frac{\tau^{2} A_{h}^{x}}{2}\right) \frac{u_{1}^{h}(x)-u_{0}^{h}(x)}{\tau}-\frac{\tau}{2}\left(f_{0}^{h}(x)-A_{h}^{x} u_{0}^{h}(x)\right) \\
=\sum_{l=1}^{n} \beta_{l}\left\{\frac{u_{\left[\lambda_{l} / \tau\right]}^{h}(x)-u_{\left[\lambda_{l} / \tau\right]-1}^{h}(x)}{\tau}+\left(\frac{\tau}{2}+\left(\lambda_{l}-\left[\frac{\lambda_{l}}{\tau}\right] \tau\right)\right) \times\left(f_{\left[\lambda_{l} / \tau\right]}^{h}(x)-A_{h}^{x} u_{\left[\lambda_{l} / \tau\right]}^{h}(x)\right)\right\} \\
+\psi^{h}(x), \quad f_{0}^{h}(x)=f^{h}(0, x), \quad x \in \widetilde{\Omega}_{h} .
\end{gathered}
$$


Theorem 2.5. Let $\tau$ and $h$ be sufficiently small positive numbers. Suppose that assumption (2.2) holds. Then, the solution of difference scheme (2.35) satisfies the following stability estimates:

$$
\begin{aligned}
& \max _{0 \leq k \leq N}\left\|u_{k}^{h}\right\|_{L_{2 h}}+\max _{0 \leq k \leq N} \sum_{r=1}^{m}\left\|\left(u_{k}^{h}\right)_{x_{r}, j_{r}}\right\|_{L_{2 h}} \\
& \quad \leq M_{1}\left[\max _{0 \leq k \leq N-1}\left\|f_{k}^{h}\right\|_{L_{2 h}}+\left\|\psi^{h}\right\|_{L_{2 h}}+\sum_{r=1}^{m}\left\|\varphi_{\overline{x_{r}}, j_{r}}^{h}\right\|_{L_{2 h}}\right] \\
& \max _{1 \leq k \leq N-1}\left\|\tau^{-2}\left(u_{k+1}^{h}-2 u_{k}^{h}+u_{k-1}^{h}\right)\right\|_{L_{2 h}}+\max _{0 \leq k \leq N} \sum_{r=1}^{m}\left\|\left(u_{k}^{h}\right)_{\overline{x_{r}} x_{r}, j_{r}}\right\|_{L_{2 h}} \\
& \quad \leq M_{1}\left[\left\|f_{0}^{h}\right\|_{L_{2 h}}+\max _{1 \leq k \leq N-1}\left\|\tau^{-1}\left(f_{k}^{h}-f_{k-1}^{h}\right)\right\|_{L_{2 h}}+\sum_{r=1}^{m}\left\|\varphi_{\overline{x_{r}}, j_{r}}^{h}\right\|_{L_{2 h}}+\sum_{r=1}^{m}\left\|\varphi_{\overline{x_{r}} x_{r}, j_{r}}\right\|_{L_{2 h}}\right] .
\end{aligned}
$$

Here, $M_{1}$ does not depend on $\tau, h, \varphi^{h}(x), \psi^{h}(x)$ and $f_{k}^{h}, 0 \leq k<N$.

The proof of Theorem 2.5 is based on abstract Theorem 2.3, symmetry properties of the operator $A_{h}^{x}$ defined by formula (2.33), and the following theorem on the coercivity inequality for the solution of the elliptic difference problem in $L_{2 h}$.

Theorem 2.6. For the solutions of the elliptic difference problem

$$
\begin{gathered}
A_{h}^{x} u^{h}(x)=\omega^{h}(x), \quad x \in \Omega_{h}, \\
u^{h}(x)=0, \quad x \in S_{h}
\end{gathered}
$$

the following coercivity inequality holds [21]:

$$
\sum_{r=1}^{m}\left\|u_{x_{r} \bar{x}_{r}, j_{r}}^{h}\right\|_{L_{2 h}} \leq M\left\|\omega^{h}\right\|_{L_{2 h}}
$$

\section{The Second Order of Accuracy Difference Scheme Generated by $A$}

Note that the difference scheme (2.1) generated by $A^{2}$ is based on the second order approximation formula for the differential equation $u^{\prime \prime}(t)+A u(t)=f(t)$ and second order of approximation formulas for nonlocal boundary conditions. Using the differential equation above, 
one can construct the second order of approximation formula for this differential equation. Thus, we obtain the following difference scheme:

$$
\begin{aligned}
& \tau^{-2}\left(u_{k+1}-2 u_{k}+u_{k-1}\right)+\frac{1}{2} A u_{k}+\frac{1}{4} A\left(u_{k+1}+u_{k-1}\right)=f_{k} \\
& f_{k}=f\left(t_{k}\right), \quad t_{k}=k \tau, 1 \leq k \leq N-1, N \tau=1, \\
& u_{0}=\sum_{m=1}^{n} \alpha_{m}\left\{u_{\left[\lambda_{m} / \tau\right]}+\tau^{-1}\left(u_{\left[\lambda_{m} / \tau\right]}-u_{\left[\lambda_{m} / \tau\right]-1}\right) \times\left(\lambda_{m}-\left[\frac{\lambda_{m}}{\tau}\right] \tau\right)\right\}+\varphi, \\
& \left(I+\frac{\tau^{2} A}{4}\right)\left[\left(I+\frac{\tau^{2} A}{4}\right) \tau^{-1}\left(u_{1}-u_{0}\right)-\frac{\tau}{2}\left(f_{0}-A u_{0}\right)\right] \\
& \quad=\sum_{k=1}^{n} \beta_{k}\left\{\tau^{-1}\left(u_{\left[\lambda_{k} / \tau\right]}-u_{\left[\lambda_{k} / \tau\right]-1}\right)+\left(\frac{\tau}{2}+\left(\lambda_{k}-\left[\frac{\lambda_{k}}{\tau}\right] \tau\right)\right) \times\left(f_{\left[\lambda_{k} / \tau\right]}-A u_{\left[\lambda_{k} / \tau\right]}\right)\right\}+\psi, \\
& f_{0}=f(0) .
\end{aligned}
$$

In exactly the same manner using the method of Section 2 one proves

Theorem 3.1. Suppose that assumption (2.2) holds and $\varphi \in D(A), \psi \in D\left(A^{1 / 2}\right)$. Then, for the solution of difference scheme (3.1) the following stability inequalities

$$
\begin{gathered}
\max _{0 \leq k \leq N}\left\|u_{k}\right\|_{H} \leq M\left\{\sum_{k=0}^{N-1}\left\|A^{-1 / 2} f_{k}\right\|_{H} \tau+\left\|A^{-1 / 2} \psi\right\|_{H}+\|\varphi\|_{H}\right\}, \\
\max _{0 \leq k \leq N}\left\|A^{1 / 2} u_{k}\right\|_{H} \leq M\left\{\sum_{k=0}^{N-1}\left\|f_{k}\right\|_{H} \tau+\left\|A^{1 / 2} \varphi\right\|_{H}+\|\varphi\|_{H}\right\}, \\
\max _{1 \leq k \leq N-1}\left\|\tau^{-2}\left(u_{k+1}-2 u_{k}+u_{k-1}\right)\right\|_{H}+\max _{1 \leq k \leq N-1}\left\|\frac{1}{2} A u_{k}+\frac{1}{4} A\left(u_{k+1}+u_{k-1}\right)\right\|_{H} \\
\leq M\left\{\sum_{k=1}^{N-1}\left\|f_{k}-f_{k-1}\right\|_{H}+\left\|f_{0}\right\|_{H}+\left\|A^{1 / 2} \psi\right\|_{H}+\|A \varphi\|_{H}\right\}
\end{gathered}
$$

hold, where $M$ does not depend on $\tau, \varphi$, $\psi$ and $f_{k}, 0 \leq k \leq N-1$.

Moreover, we can construct the difference schemes of a second order of accuracy with respect to one variable for approximate solution of nonlocal boundary value problems (2.30) and (2.34). Therefore, abstract Theorem 3.1 permits us to obtain the stability estimates for the solution of these difference schemes.

\section{Numerical Analysis}

In this section, several numerical examples are presented for the solution of problem (4.1), demonstrating the accuracy of the difference schemes. In general, we have not been able to 
determine a sharp estimate for the constants figuring in the stability inequalities. However, the numerical examples are presented for the following nonlocal boundary value problem

$$
\begin{gathered}
\frac{\partial^{2} u(t, x)}{\partial t^{2}}-\frac{\partial^{2} u(t, x)}{\partial x^{2}}+u(t, x)=\left(6 t+2 t^{3}\right) \sin x, \quad 0<t<1,0<x<\pi, \\
u(0, x)=\frac{1}{4} u(1, x)-\frac{1}{4} u\left(\frac{1}{2}, x\right)-\frac{7}{32} \sin x, \quad 0 \leq x \leq \pi, \\
u_{t}(0, x)=\frac{1}{4} u_{t}(1, x)-\frac{1}{4} u_{t}\left(\frac{1}{2}, x\right)-\frac{9}{16} \sin x, \quad 0 \leq x \leq \pi, \\
u(t, 0)=u(t, \pi)=0, \quad 0 \leq t \leq 1 .
\end{gathered}
$$

The exact solution of this problem is $u(t, x)=t^{3} \sin x$.

We consider the set $[0,1]_{\tau} \times[0, \pi]_{h}$ of a family of grid points depending on the small parameters $\tau$ and $h:[0,1]_{\tau} \times[0, \pi]_{h}=\left\{\left(t_{k}, x_{n}\right): t_{k}=k \tau, 0 \leq k \leq N, N \tau=1, x_{n}=\right.$ $n h, 0 \leq n \leq M, M h=\pi\}$. For the approximate solution of problem (4.1), we have applied the first order and the two different types of second orders of accuracy difference schemes, respectively. Solving these difference equations we have applied a procedure of modified Gauss elimination method with respect to $n$ with matrix coefficients.

First let us consider the first order of accuracy in time difference scheme (1.4) for the approximate solution of the Cauchy problem (4.1). Using difference scheme (1.4), we obtain

$$
\begin{gathered}
\frac{u_{n}^{k+1}-2 u_{n}^{k}+u_{n}^{k-1}}{\tau^{2}}-\frac{u_{n+1}^{k+1}-2 u_{n}^{k+1}+u_{n-1}^{k+1}}{h^{2}}+u_{n}^{k+1}=f\left(t_{k+1}, x_{n}\right), t_{k}=k \tau, \\
1 \leq k \leq N-1, \quad N \tau=1, \quad x_{n}=n h, \quad 1 \leq n \leq M-1, \quad M h=\pi, \\
u_{n}^{0}=\frac{1}{4} u_{n}^{N}-\frac{1}{4} u_{n}^{(N / 2)+1}-\frac{7}{32} \sin x, \quad 1 \leq n \leq M-1, \\
\tau^{-1}\left(u_{n}^{1}-u_{n}^{0}\right)=\frac{1}{4} \tau^{-1}\left(u_{n}^{N}-u_{n}^{N-1}\right)-\frac{1}{4} \tau^{-1}\left(u_{n}^{(N / 2)+1}-u_{n}^{N / 2}\right)-\frac{9}{16} \sin x, \\
x_{n}=n h, \quad 1 \leq n \leq M-1, \quad u_{0}^{k}=u_{M}^{k}=0, \quad 0 \leq k \leq N,
\end{gathered}
$$

for the approximate solution of Cauchy problem (4.1). We have $(N+1) \times(N+1)$ system of linear equation in (4.2) and we can write them in the matrix form as

$$
\begin{gathered}
A U_{n+1}+B U_{n}+C U_{n-1}=D \varphi_{n}, \quad 1 \leq n \leq M-1, \\
U_{0}=\tilde{0}, \quad U_{M}=\widetilde{0},
\end{gathered}
$$

where

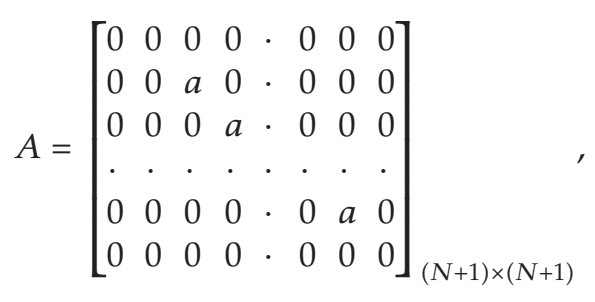




$$
\begin{aligned}
& B=\left[\begin{array}{ccccccccccccc}
1 & 0 & 0 & 0 & 0 & \cdot & \frac{1}{4} & 0 & \cdot & 0 & 0 & 0 & -\frac{1}{4} \\
b & c & d & 0 & 0 & \cdot & 0 & 0 & \cdot & 0 & 0 & 0 & 0 \\
0 & b & c & d & 0 & \cdot & 0 & 0 & \cdot & 0 & 0 & 0 & 0 \\
0 & 0 & b & c & d & \cdot & 0 & 0 & \cdot & 0 & 0 & 0 & 0 \\
\cdot & \cdot & \cdot & \cdot & \cdot & \cdot & \cdot & \cdot & \cdot & \cdot & \cdot & \cdot & \cdot \\
0 & 0 & 0 & 0 & 0 & \cdot & 0 & 0 & \cdot & 0 & 0 & 0 & 0 \\
0 & 0 & 0 & 0 & 0 & \cdot & 0 & 0 & \cdot & b & c & d & 0 \\
0 & 0 & 0 & 0 & 0 & \cdot & 0 & 0 & \cdot & 0 & b & c & d \\
-1 & 1 & 0 & 0 & 0 & \cdot & -\frac{1}{4} & \frac{1}{4} & \cdot & 0 & 0 & \frac{1}{4} & -\frac{1}{4}
\end{array}\right]_{(N+1) \times(N+1)} \\
& C=A, \quad D=\left[\begin{array}{llll}
1 & 0 & \cdot & 0 \\
0 & 1 & \cdot & 0 \\
\cdot & \cdot & \cdot & \cdot \\
0 & 0 & \cdot & 1
\end{array}\right]_{(N+1) \times(N+1)} \text {, } \\
& \varphi_{n}^{k}=\left[\begin{array}{c}
\varphi_{n}^{0} \\
\varphi_{n}^{1} \\
\vdots \\
\varphi_{n}^{N}
\end{array}\right]_{(N+1) \times 1} \quad, \quad 0 \leq k \leq N \\
& \varphi_{n}^{0}=-\frac{7}{32} \sin \left(x_{n}\right), \quad \varphi_{n}^{N}=-\frac{9}{16} \sin \left(x_{n}\right), \\
& \varphi_{n}^{k}=\left\{\begin{array}{l}
\left(6\left(t_{k+1}\right)+2\left(t_{k+1}\right)^{3}\right) \sin \left(x_{n}\right), \\
f\left(t_{k+1}, x_{n}\right), \quad 1 \leq k \leq N-1,
\end{array}\right. \\
& U_{s}^{k}=\left[\begin{array}{c}
u_{s}^{0} \\
u_{s}^{1} \\
\vdots \\
u_{s}^{N}
\end{array}\right]_{(N+1) \times 1}, \quad 0 \leq k \leq N, s=n \pm 1, n
\end{aligned}
$$

Here,

$$
a=-\frac{1}{h^{2}}, \quad b=\frac{1}{\tau^{2}}, \quad c=\frac{-2}{\tau^{2}}, \quad d=\frac{1}{\tau^{2}}+\frac{2}{h^{2}}+1 .
$$

For the solution of difference equation (4.2), we have applied the modified Gauss elimination method. Therefore, we seek a solution of the matrix equation by using the following iteration formula:

$$
u_{n}=\alpha_{n+1} u_{n+1}+\beta_{n+1}, \quad n=M-1, \ldots, 2,1,0,
$$


where $\alpha_{j}, \beta_{j}(j=1, \ldots, M)$ are $(N+1) \times(N+1)$ square matrices and $\gamma_{j}$ are $(N+1) \times 1$ column matrices. Now, we obtain formula of $\alpha_{n+1}, \beta_{n+1}$,

$$
\begin{gathered}
\alpha_{n+1}=-\left(B+C \alpha_{n}\right)^{-1} A \\
\beta_{n+1}=\left(B+C \alpha_{n}\right)^{-1}\left(D \varphi_{n}-C \beta_{n}\right), \quad n=1,2,3, \ldots M-1 .
\end{gathered}
$$

Note that

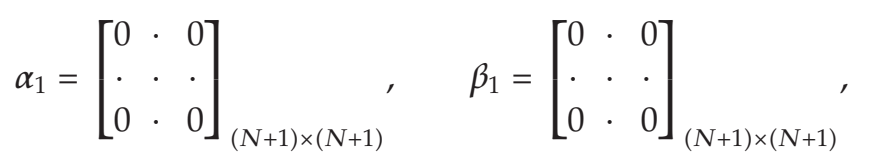

and $U_{M}=\tilde{0}$.

Thus, using the formulas and matrices above we obtain the difference scheme first order of accuracy in $t$ and second order of accuracy in $x$ for approximate solution of nonlocal boundary value problem (4.1).

Second, let us consider the second order of accuracy in time implicit difference scheme (3.1) for the approximate solution of problem (4.1). Using difference scheme (3.1), we obtain

$$
\begin{gathered}
\frac{U_{n}^{k+1}-2 U_{n}^{k}+U_{n}^{k-1}}{\tau^{2}}-\frac{U_{n+1}^{k}-2 U_{n}^{k}+U_{n-1}^{k}}{2 h^{2}}-\frac{U_{n+1}^{k+1}-2 U_{n}^{k+1}+U_{n-1}^{k+1}}{4 h^{2}}-\frac{U_{n+1}^{k-1}-2 U_{n}^{k-1}+U_{n-1}^{k-1}}{4 h^{2}} \\
=\left(6\left(t_{k}\right)+2\left(t_{k}\right)^{3}\right) \sin \left(x_{n}\right), \quad t_{k}=k \tau, 1 \leq k \leq N-1, N \tau=1, \\
x_{n}=n h, \quad 1 \leq n \leq M-1, \quad M h=\pi \\
\left(I+\frac{\tau^{2} A_{h}^{x}}{4}\right)\left[\left(I+\frac{\tau^{2} A_{h}^{x}}{4}\right)\left(u_{1}^{h}\left(x_{n}\right)-u_{0}^{h}\left(x_{n}\right)\right)+\frac{\tau}{2}\left(f^{h}\left(0, x_{n}\right)-A_{h}^{x} u_{0}^{h}\left(x_{n}\right)\right)\right] \\
=\frac{1}{4}(2 \tau)^{-1}\left(-u_{N-2}^{h}\left(x_{n}\right)+4 u_{N-1}^{h}\left(x_{n}\right)-3 u_{N}^{h}\left(x_{n}\right)\right) \\
-\frac{1}{4}(2 \tau)^{-1}\left(-3 u_{(N / 2)+1}^{h}\left(x_{n}\right)+4 u_{(N / 2)+1}^{h}\left(x_{n}\right)-u_{(N / 2)+3}^{h}\left(x_{n}\right)\right)-\frac{9}{16} \sin \left(x_{n}\right), \quad 1 \leq n \leq M-1, \\
u_{0}^{k}=u_{M}^{k}=0, \quad 0 \leq k \leq N,
\end{gathered}
$$


for the approximate solution of problem (4.1). We have again the same linear system (4.3); therefore, we use exactly the same method that we have used for the solution of difference equation (4.2), but matrices are different as follows:

$$
\begin{aligned}
& A=\left[\begin{array}{cccccccc}
0 & 0 & 0 & 0 & \cdot & 0 & 0 & 0 \\
z & w & z & 0 & \cdot & 0 & 0 & 0 \\
0 & z & w & z & \cdot & 0 & 0 & 0 \\
\cdot & \cdot & \cdot & \cdot & \cdot & \cdot & \cdot & \cdot \\
0 & 0 & 0 & 0 & \cdot & z & w & z \\
0 & 0 & 0 & 0 & \cdot & 0 & 0 & 0
\end{array}\right]_{(N+1) \times(N+1)} \\
& B=\left[\begin{array}{cccccccccccc}
1 & 0 & 0 & 0 & \cdot & \frac{1}{4} & 0 & 0 & \cdot & 0 & 0 & -\frac{1}{4} \\
b & c & d & 0 & \cdot & 0 & 0 & 0 & \cdot & 0 & 0 & 0 \\
0 & b & c & d \cdot & 0 & 0 & 0 & \cdot & 0 & 0 & 0 \\
\cdot & \cdot & \cdot & \cdot & \cdot & \cdot & \cdot & \cdot & \cdot & \cdot & \cdot & \cdot \\
0 & 0 & 0 & 0 & \cdot & 0 & 0 & 0 & \cdot & 0 & 0 & 0 \\
0 & 0 & 0 & 0 & \cdot & 0 & 0 & 0 & \cdot & c & d & 0 \\
0 & 0 & 0 & 0 & \cdot & 0 & 0 & 0 & \cdot & b & c & d \\
-\frac{3}{2} \tau & \frac{4}{2} \tau & -\frac{1}{2} \tau & 0 & \cdot & -\frac{3}{8} \tau & \frac{4}{8} \tau & -\frac{1}{8} \tau & \cdot & -\frac{1}{8} \tau & \frac{4}{8} \tau & -\frac{3}{8} \tau
\end{array}\right]_{(N+1) \times(N+1)} \\
& C=A, \quad D=\left[\begin{array}{cccc}
1 & 0 & \cdot & 0 \\
0 & 1 & \cdot & 0 \\
\cdot & \cdot & \cdot & \cdot \\
0 & 0 & \cdot & 1
\end{array}\right]_{(N+1) \times(N+1)}
\end{aligned}
$$

Here,

$$
x=\frac{1}{\tau^{2}}+\frac{1}{2 h^{2}}+\frac{1}{4}, \quad y=-\frac{2}{\tau^{2}}+\frac{1}{h^{2}}+\frac{1}{2}, \quad z=\frac{-1}{4 h^{2}}, \quad w=-\frac{1}{2 h^{2}} .
$$

Thus, using the above matrices we obtain the difference scheme second order of accuracy in $t$ and $x$ for approximate solution of nonlocal boundary value problem (4.1).

Next, let us consider the second order of accuracy in time implicit difference scheme (3.1) for the approximate solution of problem (4.1). Using difference scheme (3.1), we obtain

$$
\begin{aligned}
& \frac{u_{n}^{k+1}-2}{\tau^{2}}+u_{n}^{k-1} \\
& \quad-\frac{u_{n+1}^{k}-2 u_{n}^{k}+u_{n-1}^{k}}{h^{2}}+u_{n}^{k} \\
& \quad \frac{\tau^{2}}{4}\left(\frac{u_{n+2}^{k+1}-4 u_{n+1}^{k+1}+6 u_{n}^{k+1}-4 u_{n-1}^{k+1}+u_{n-2}^{k+1}}{h^{4}}-2 \frac{u_{n+1}^{k+1}-2 u_{n}^{k+1}+u_{n-1}^{k+1}}{h^{2}}\right) \\
& =\left(6\left(t_{k}\right)+2\left(t_{k}\right)^{3}\right) \sin \left(x_{n}\right),
\end{aligned}
$$




$$
\begin{gathered}
t_{k}=k \tau, \quad 1 \leq k \leq N-1, \quad N \tau=1, \quad x_{n}=n h, \quad 1 \leq n \leq M-1, \quad M h=\pi, \\
u_{n}^{0}=\frac{1}{4} u^{N}-\frac{1}{4} u^{(N / 2)+1}-\frac{7}{32} \sin x, \quad 1 \leq n \leq M-1, \\
\left(I+\frac{\tau^{2} A_{h}^{x}}{4}\right)\left[\left(I+\frac{\tau^{2} A_{h}^{x}}{4}\right)\left(u_{1}^{h}\left(x_{n}\right)-u_{0}^{h}\left(x_{n}\right)\right)+\frac{\tau}{2}\left(f^{h}\left(0, x_{n}\right)-A_{h}^{x} u_{0}^{h}\left(x_{n}\right)\right)\right] \\
=\frac{1}{4}(2 \tau)^{-1}\left(-u_{N-2}^{h}\left(x_{n}\right)+4 u_{N-1}^{h}\left(x_{n}\right)-3 u_{N}^{h}\left(x_{n}\right)\right) \\
-\frac{1}{4}(2 \tau)^{-1}\left(-3 u_{(N / 2)+1}^{h}\left(x_{n}\right)+4 u_{(N / 2)+1}^{h}\left(x_{n}\right)-u_{(N / 2)+3}^{h}\left(x_{n}\right)\right) \\
-\frac{9}{16} \sin \left(x_{n}\right), \quad 1 \leq n \leq M-1, \\
u_{0}^{k}=u_{M}^{k}=0, \quad 0 \leq k \leq N . \\
u_{1}^{k}=\frac{4}{5} u_{2}^{k}-\frac{1}{5} u_{3}^{k}, \quad u_{M-1}^{k}=\frac{4}{5} u_{M-2}^{k}-\frac{1}{5} u_{M-3}^{k}, \quad 0 \leq k \leq N,
\end{gathered}
$$

for the approximate solution of problem (4.1). We have $(N+1) \times(N+1)$ system of linear equation in (4.12) and we can write in the matrix form as

$$
\begin{gathered}
A U_{n+2}+B U_{n+1}+C U_{n}+D U_{n-1}+E U_{n-2}=R \varphi_{n}, \quad 2 \leq n \leq M-2, \\
U_{0}=U_{M}=\tilde{0}, \quad U_{3}^{k}=4 U_{2}^{k}-5 U_{1}^{k}, \quad U_{M-3}^{k}=4 U_{M-2}^{k}-5 U_{M-1}^{k}, \quad 0 \leq k \leq N,
\end{gathered}
$$

where

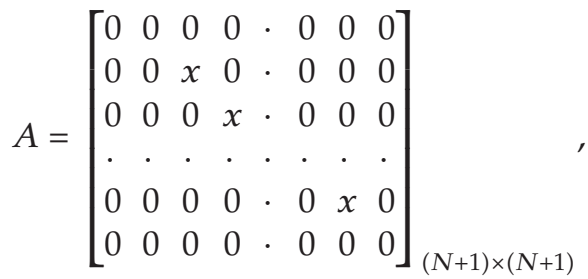

$$
\begin{aligned}
& B=\left[\begin{array}{cccccccc}
0 & 0 & 0 & 0 & \cdot & 0 & 0 & 0 \\
0 & w & y & 0 & \cdot & 0 & 0 & 0 \\
0 & 0 & w & y & \cdot & 0 & 0 & 0 \\
\cdot & \cdot & \cdot & \cdot & \cdot & \cdot & \cdot & \cdot \\
0 & 0 & 0 & 0 & \cdot & w & y & 0 \\
0 & 0 & 0 & 0 & \cdot & 0 & 0 & 0
\end{array}\right]_{(N+1) \times(N+1)}
\end{aligned}
$$


Abstract and Applied Analysis

$$
\begin{gathered}
C=\left[\begin{array}{cccccccccccc}
1 & 0 & 0 & 0 & \cdot & \frac{1}{4} & 0 & 0 & \cdot & 0 & 0 & -\frac{1}{4} \\
v & t & z & 0 & \cdot & 0 & 0 & 0 & \cdot & 0 & 0 & 0 \\
0 & v & t & z & 0 & 0 & 0 & \cdot & 0 & 0 & 0 \\
\cdot & \cdot & \cdot & \cdot & \cdot & \cdot & \cdot & \cdot & \cdot & \cdot & \cdot & \cdot \\
0 & 0 & 0 & 0 & \cdot & 0 & 0 & 0 & \cdot & 0 & 0 & 0 \\
0 & 0 & 0 & 0 & \cdot & 0 & 0 & 0 & \cdot & t & z & 0 \\
0 & 0 & 0 & 0 & \cdot & 0 & 0 & 0 & \cdot & v & t & z \\
3 & 4 & \frac{4}{2} \tau & -\frac{1}{2} \tau & 0 & \cdot & -\frac{3}{8} \tau & \frac{4}{8} \tau & -\frac{1}{8} \tau \cdot c & -\frac{1}{8} \tau & \frac{4}{8} \tau & -\frac{3}{8} \tau
\end{array}\right]_{(N+1) \times(N+1)} \\
D=B, \quad E=A,\left[\begin{array}{cccc}
1 & 0 & \cdot & 0 \\
0 & 1 & \cdot & 0 \\
\cdot & \cdot & \cdot & \cdot \\
0 & 0 & \cdot & 1
\end{array}\right]_{(N+1) \times(N+1)}, \\
U_{s}^{k}=\left[\begin{array}{c}
u_{s}^{0} \\
u_{s}^{1} \\
\vdots \\
u_{s}^{N}
\end{array}\right]_{(N+1) \times 1}, \quad 0 \leq k \leq N, s=n \pm 2, n \pm 1, n .
\end{gathered}
$$

Here,

$$
\begin{gathered}
x=\frac{\tau^{2}}{4 h^{4}}, \quad y=-\frac{\tau^{2}}{h^{4}}, \quad z=\frac{1}{\tau^{2}}+\frac{3 \tau^{2}}{2 h^{4}}, \quad t=\frac{-2}{\tau^{2}}, \\
d=\frac{1}{\tau^{2}}+\frac{2}{h^{2}}+1, \quad v=\frac{1}{\tau^{2}}, \quad w=-\frac{1}{h^{2}} .
\end{gathered}
$$

For the solution of the linear system (4.13), we use the modified variant Gauss elimination method and seek a solution of the matrix equation by the following form:

$$
u_{n}=\alpha_{n+1} u_{n+1}+\beta_{n+1} u_{n+2}+\gamma_{n+1}, \quad n=M-2, \ldots, 2,1,0,
$$

where $\alpha_{j}, \beta_{j}(j=1: M-1)$ are $(N+1) \times(N+1)$ square matrices and $\gamma_{j}$-s are $(N+1) \times 1$ column matrices. We obtain the following formulas of $\alpha_{n+1}, \beta_{n+1}, \gamma_{n+1}$ from linear system (4.13) by using formula (4.16):

$$
\begin{aligned}
& \alpha_{n+1}= {\left[C+D \alpha_{n}+E \alpha_{n-1} \alpha_{n}+E \beta_{n-1}\right]^{-1} } \\
& \times\left[-B-D \beta_{n}-E \alpha_{n-1} \beta_{n}\right], \\
& \beta_{n+1}=- {\left[C+D \alpha_{n}+E \alpha_{n-1} \alpha_{n}+E \beta_{n-1}\right]^{-1} A, } \\
& \gamma_{n+1}=\left[C+D \alpha_{n}+E \alpha_{n-1} \alpha_{n}+E \beta_{n-1}\right]^{-1} \\
& \times\left[R \varphi_{n}-D \gamma_{n}+E \alpha_{n-1} \gamma_{n}+E \gamma_{n-1}\right] .
\end{aligned}
$$


We have $\alpha_{1}, \beta_{1}, \gamma_{1}, \alpha_{2}, \beta_{2}, \gamma_{2}$ :

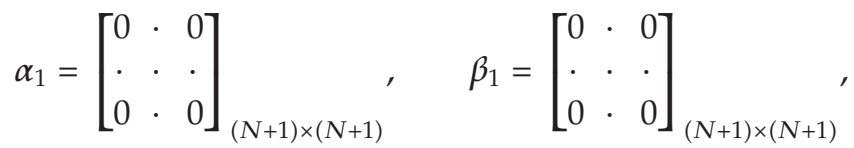

$$
\begin{aligned}
& r_{1}=\left[\begin{array}{c}
0 \\
0 \\
\vdots \\
0
\end{array}\right]_{(N+1) \times(1)}, \quad r_{2}=\left[\begin{array}{c}
0 \\
0 \\
\vdots \\
0
\end{array}\right]_{(N+1) \times(1)}, \\
& \alpha_{2}=\left[\begin{array}{cccc}
\frac{4}{5} & 0 & \cdot & 0 \\
0 & \frac{4}{5} \cdot & 0 \\
\cdot & \cdot & \cdot & \cdot \\
0 & 0 & \cdot & \frac{4}{5}
\end{array}\right]_{(N+1) \times(N+1)}, \quad \beta_{2}=\left[\begin{array}{cccc}
-\frac{1}{5} & 0 & \cdot & 0 \\
0 & -\frac{1}{5} & \cdot & 0 \\
\cdot & \cdot & \cdot & \cdot \\
0 & 0 & \cdot & -\frac{1}{5}
\end{array}\right]_{(N+1) \times(N+1)} \\
& U_{M}=\tilde{0}, \quad U_{M-1}=\left[\left(\beta_{M-2}+5 I\right)-\left(4 I-\alpha_{M-2}\right) \alpha_{M-1}\right]^{-1}\left[\left(4 I-\alpha_{M-2}\right) \gamma_{M-1}-\gamma_{M-2}\right] \text {. }
\end{aligned}
$$

Thus, using the above matrices we obtain the difference scheme second order of accuracy in $t$ and $x$ for approximate solution of nonlocal boundary value problem (4.1).

Matlab is a programming language for numeric scientific computation. One of its characteristic features is the use of matrices as the only data type. Therefore, the implementations of numerical examples are carried out by Matlab.

Now, we will give some numerical results for the solutions of (4.2), (4.9), and (4.12) for different $M=N$ values where $N$ and $M$ are the step numbers for the time and space variables, respectively. Note that the grid step numbers $N$ and $M$ in the given examples are chosen equal for clarity and this is not necessary for the stability and solutions of the difference schemes.

The errors are computed by the following formula:

$$
E_{M}^{N}=\max _{1 \leq k \leq M-1}\left(\sum_{k=1}^{N-1}\left|u\left(t_{k}, x_{n}\right)-u_{n}^{k}\right|^{2} h\right)^{1 / 2}
$$

Here, $u\left(t_{k}, x_{n}\right)$ represents the exact solution and $u_{n}^{k}$ represents the approximate solution at $\left(t_{k}, x_{n}\right)$. The errors and the related CPU times are presented in Tables 1 and 2, respectively, for $N=M=20,30,40,80,300$, and 500. The implementations are carried out by MATLAB 7.1 software package and obtained by a PC System 64 bit, Pentium (R) Core(TM) i5 CPU, 3.20 $6 \mathrm{~Hz}, 3.19 \mathrm{~Hz}, 4000 \mathrm{Mb}$ of RAM.

Let us denote the first order of accuracy difference scheme (4.2) as $F$, the second order of accuracy difference scheme generated by three points (4.9) as $S 1$, and the second order of accuracy difference scheme generated by five points (4.12) as $S 2$.

In order to get accurate results, CPU times are recorded by running each program 100 times for small values $N=M=20,30,40,80$ and taking the average of the elapsed time. 
Table 1: Comparison of errors for approximate solutions.

\begin{tabular}{lcccccc}
\hline Difference Scheme & 20 & 30 & 40 & 80 & 300 & 500 \\
\hline$F$ & 0.0494 & 0.0324 & 0.0241 & 0.0119 & 0.0032 & 0.0019 \\
$S 1$ & 0.0020 & 0.0008 & 0.0004918 & 0.0001230 & 0.0000087 & 0.0000031 \\
$S 2$ & 0.0031 & 0.0015 & 0.0007875 & 0.0001971 & 0.0000140 & \\
\hline
\end{tabular}

Table 2: CPU times.

\begin{tabular}{lcccccc}
\hline Difference Scheme & 20 & 30 & 40 & 80 & 300 & 500 \\
\hline$F$ & 0.0080 & 0.0261 & 0.0542 & 0.4518 & 69.384 & 545.411 \\
$S 1$ & 0.0086 & 0.0267 & 0.0572 & 0.4631 & 69.933 & 532.852 \\
$S 2$ & 0.0112 & 0.0441 & 0.1042 & 0.9842 & 169.880 & \\
\hline
\end{tabular}

The following conclusions can be noted for the comparison of the numerical results presented in the tables above.

(i) In the tables, it is noted that almost the same accuracy is achieved by $S 1$ with data error $=0.0020, N=20$ and by $F$ with data error $=0.0019, N=500$ in different CPU times; $545 \mathrm{~s}$ and $0.0086 \mathrm{~s}$, respectively. This means the use of the difference scheme $S 1$ accelerates the computation with a ratio of more than $545 / 0.0086 \cong 63372$ times, that is, $S 1$ is considerably faster than $F$.

(ii) It is also noted that almost the same accuracy is achieved by the difference scheme $S 2$ with data error $=0.0031, N=20$, and by the difference scheme $F$ with data error $=0.0032, N=300$ in different CPU times; $0.0112 \mathrm{~s}$ and $69 \mathrm{~s}$, respectively. Thus, the use of the difference scheme $S 2$ accelerates the computation with a ratio of more than $69 / 0.0112 \cong 6160$ times, that is, $S 2$ is considerably faster than $F$.

(iii) When we consider almost the same CPU times for $F$ and $S 1$ as $0.0080 \mathrm{~s} \cong$ $0.0086 \mathrm{~s}, F$ computes the solution with an error $=0.0494$, for $N=20$, where the difference scheme $S 1$ computes the solution with an error $=0.0020$, which is almost 25 times smaller error than the computation error of $F$. Namely, $S 1$ yields 25 times more accurate results than $F$. For $S 2$, this ratio reduces to $0.0112 / 0.0080 \cong 1.4$ times.

(iv) While both types of the second order difference schemes reach approximately the same accuracy, the CPU time for the difference scheme $S 2$ is always greater than S1.

(v) The CPU times of difference schemes $F, S 1$, and $S 2$ recorder for $N \geq 100$ exceed ones. Matlab gives "out of memory" error for $S 2$ with values $N=500$ which means the memory of the computer is not enough. It is not necessary to have results for $S 2$ when $N=500$, since it is obvious that CPU time of $S 2$ is more than that of $F$ and S1.

(vi) It is observed from the tables that for larger $N$ values the numerical results become approximately the same for each difference scheme in the reliable range of the CPU times. This indicates that the approximation made for the solution of problem (4.1) is valid.

In conclusion, the second order difference schemes are much more accurate than the first order difference scheme, and the second order difference scheme generated by three points is more convenient than the second order difference scheme generated by five points 
when considering the CPU times and the error levels. Comparing with many other numerical methods, our method is not based on the relationship between the grid step sizes of time and space variables (see [25-30] and the references therein).

\section{Acknowledgment}

The authors would like to thank Professor P. E. Sobolevskii for his helpful suggestions to the improvement of this paper.

\section{References}

[1] H. Lamb, Hydrodynamics, Cambridge Mathematical Library, Cambridge University Press, Cambridge, UK, 6th edition, 1993.

[2] J. Lighthill, Waves in Fluids, Cambridge University Press, Cambridge, UK, 1978.

[3] J. A. Hudson, The Excitation and Propagation of Elastic Waves, Cambridge University Press, Cambridge, UK, 1980.

[4] D. S. Jones, Acoustic and Electromagnetic Waves, Oxford Science Publications, The Clarendon Press Oxford University Press, New York, NY, USA, 1986.

[5] A. Taflove, Computational Electrodynamics, Artech House, Boston, Mass, USA, 1995.

[6] A. Ashyralyev and N. Aggez, "A note on the difference schemes of the nonlocal boundary value problems for hyperbolic equations," Numerical Functional Analysis and Optimization, vol. 25, no. 5-6, pp. 439-462, 2004.

[7] A. Ashyralyev and O. Yildirim, "Second order of accuracy stable difference schemes for hyperbolic problems subject to nonlocal conditions with self-adjoint operator," in Proceedings of the International Conference on Numerical Analysis and Applied Mathematics: Numerical Analysis and Applied Mathematics (ICNAAM '11), vol. 1389 of AIP Conference Proceedings, pp. 597-600, 2011.

[8] A. Ashyralyev and O. Yildirim, "On multipoint nonlocal boundary value problems for hyperbolic differential and difference equations," Taiwanese Journal of Mathematics, vol. 14, no. 1, pp. 165-194, 2010.

[9] M. E. Koksal, "Recent developments on operator-difference schemes for solving nonlocal BVPs for the wave equation," Discrete Dynamics in Nature and Society, vol. 2011, Article ID 210261, 14 pages, 2011.

[10] A. Ashyralyev, M. E. Koksal, and R. P. Agarwal, "A difference scheme for Cauchy problem for the hyperbolic equation with self-adjoint operator," Mathematical and Computer Modelling, vol. 52, no. 1-2, pp. 409-424, 2010.

[11] M. Sapagovas, "On stability of the finite difference schemes for a parabolic equations with nonlocal condition," Journal of Computer Applied Mathematics, vol. 1, no. 1, pp. 89-98, 2003.

[12] M. Dehghan, "Fully implicit finite differences methods for two-dimensional diffusion with a nonlocal boundary condition," "Journal of Computational and Applied Mathematics, vol. 24, pp. 1-8, 2007.

[13] N. Gordeziani, P. Natalini, and P. E. Ricci, "Finite-difference methods for solution of nonlocal boundary value problems," Computers \& Mathematics with Applications, vol. 106, no. 2, pp. 255-269, 1999.

[14] D. Gordezani, H. Meladze, and G. Avalishvili, "On one class of nonlocal in time problems for firstorder evolution equations," Zhurnal Obchyslyuval'no Ta Prykladno I Matematyky, vol. 88, no. 1, pp. 66-78, 2003.

[15] J. Martín-Vaquero, A. Queiruga-Dios, and A. H. Encinas, "Numerical algorithms for diffusionreaction problems with non-classical conditions," Applied Mathematics and Computation, vol. 218, no. 9, pp. 5487-5495, 2012.

[16] A. Ashyralyev and O. Gercek, "On second order of accuracy difference scheme of the approximate solution of nonlocal elliptic-parabolic problems," Abstract and Applied Analysis, vol. 2010, Article ID 705172, 17 pages, 2010.

[17] A. Ashyralyev and P. E. Sobolevskii, "Two new approaches for construction of the high order of accuracy difference schemes for hyperbolic differential equations," Discrete Dynamics in Nature and Society, no. 2, pp. 183-213, 2005. 
[18] A. Ashyralyev and P. E. Sobolevskii, "A note on the difference schemes for hyperbolic equations," Abstract and Applied Analysis, vol. 6, no. 2, pp. 63-70, 2001.

[19] P. E. Sobolevskii and L. M. Chebotaryeva, "Approximate solution by method of lines of the Cauchy problem for an abstract hyperbolic equations," Izvestiya Vysshikh Uchebnykh Zavedenii. Matematika, vol. 5, no. 1, pp. 103-116, 1977.

[20] A. Ashyralyev and P. E. Sobolevskii, New Difference Schemes for Partial Differential Equations, vol. 148 of Operator Theory: Advances and Applications, Birkhäuser, Basel, Switzerland, 2004.

[21] P. E. Sobolevskii, Difference Methods for the Approximate Solution of Differential Equations, Izdatelstvo Voronezhskogo Gosud Universiteta, Voronezh, Russia, 1975.

[22] A. Ashyralyev, G. Judakova, and P. E. Sobolevskii, "A note on the difference schemes for hyperbolicelliptic equations," Abstract and Applied Analysis, vol. 2006, Article ID 14816, 13 pages, 2006.

[23] A. Ashyralyev and Y. Ozdemir, "On stable implicit difference scheme for hyperbolic-parabolic equations in a Hilbert space," Numerical Methods for Partial Differential Equations, vol. 25, no. 5, pp. 1100-1118, 2009.

[24] A. A. Samarskii, V. I. Korzyuk, S. V. Lemeshevsky, and P. P. Matus, "Finite-difference methods for problem of conjugation of hyperbolic and parabolic equations," Mathematical Models $\mathcal{E}$ Methods in Applied Sciences, vol. 10, no. 3, pp. 361-377, 2000.

[25] M. Ciment and S. H. Leventhal, "A note on the operator compact implicit method for the wave equation," Mathematics of Computation, vol. 32, no. 141, pp. 143-147, 1978.

[26] E. H. Twizell, "An explicit difference method for the wave equation with extended stability range," BIT, vol. 19, no. 3, pp. 378-383, 1979.

[27] P. D. Lax and B. Wendroff, "Difference schemes for hyperbolic equations with high order of accuracy," Communications on Pure and Applied Mathematics, vol. 17, pp. 381-398, 1964.

[28] R. K. Mohanty, M. K. Jain, and K. George, "On the use of high order difference methods for the system of one space second order nonlinear hyperbolic equations with variable coefficients," Journal of Computational and Applied Mathematics, vol. 72, no. 2, pp. 421-431, 1996.

[29] S. Piskarev, "Approximation of holomorphic semigroups," Tartu Riikliku Ülikooli Toimetised, no. 492, pp. 3-14, 1979.

[30] S. I. Piskarëv, "Stability of difference schemes in Cauchy problems with almost periodic solutions," Differentsial nye Uravneniya, vol. 20, no. 4, pp. 689-695, 1984.

[31] M. I. Gil', Difference Equations in Normed Spaces, vol. 206 of North-Holland Mathematics Studies, Elsevier Science B.V., Amsterdam, The Netherlands, 2007.

[32] A. R. Mitchell and D. F. Griffiths, The Finite Difference Method in Partial Differential Equations, John Wiley \& Sons, Chichester, UK, 1980.

[33] H. O. Fattorini, Second Order Linear Differential Equations in Banach Spaces, vol. 108 of North-Holland Mathematics Studies, North-Holland, Amsterdam, The Netherlands, 1985.

[34] A. A. Samarskii, "Some problems of the theory of differential equations," Differentsial' nye Uravneniya, vol. 16, pp. 1221-1228, 1980.

[35] B. Gustafson, H. O. Kreiss, and J. Oliger, Time Dependent Problems and Difference Methods, John Wiley \& Sons, New York, NY, USA, 1995.

[36] T. E. Simos and P. S. Williams, "A finite-difference method for the numerical solution of the Schrödinger equation," Journal of Computational and Applied Mathematics, vol. 79, no. 2, pp. 189-205, 1997.

[37] S. G. Krein, Linear Differential Equations in a Banach Space, Nauka, Moscow, Russia, 1966.

[38] S. Piskarev and S.-Y. Shaw, "On certain operator families related to cosine operator functions," Taiwanese Journal of Mathematics, vol. 1, no. 4, pp. 527-546, 1997. 


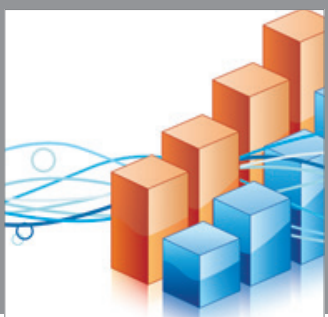

Advances in

Operations Research

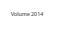

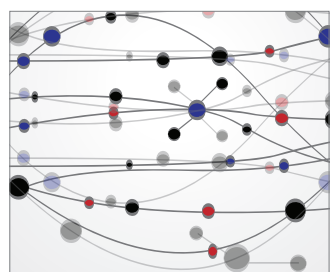

\section{The Scientific} World Journal
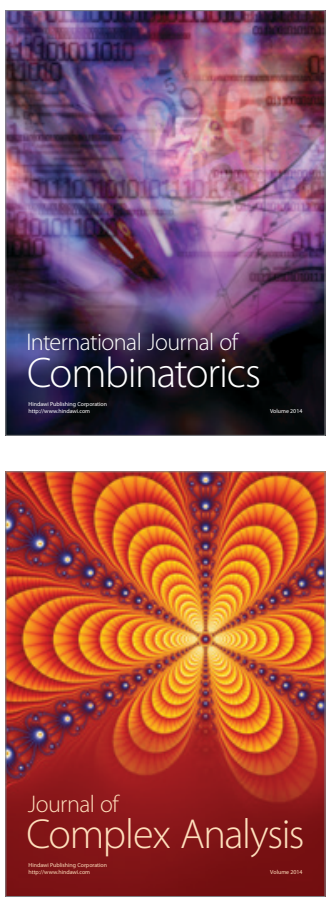

International Journal of

Mathematics and

Mathematical

Sciences
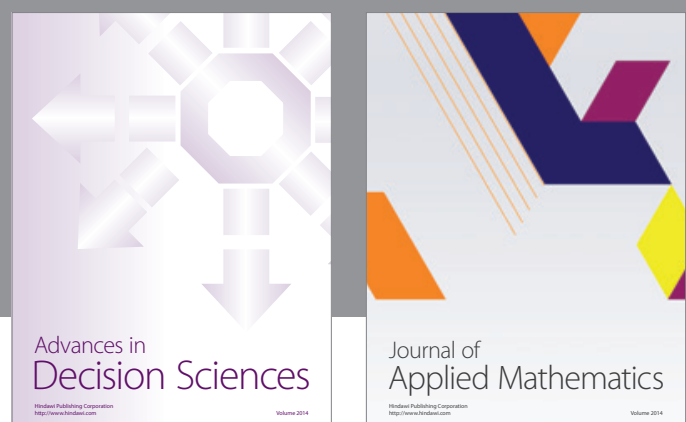

Journal of

Applied Mathematics
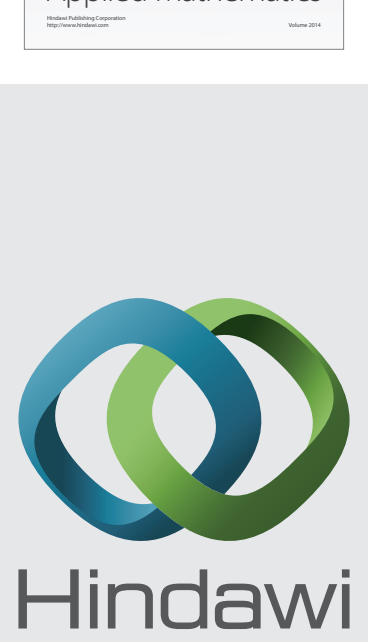

Submit your manuscripts at http://www.hindawi.com
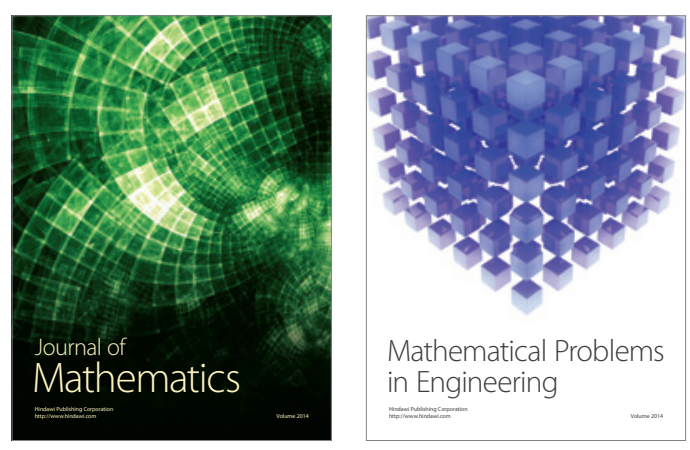

Mathematical Problems in Engineering
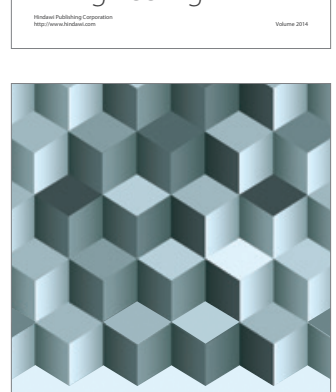

Journal of

Function Spaces
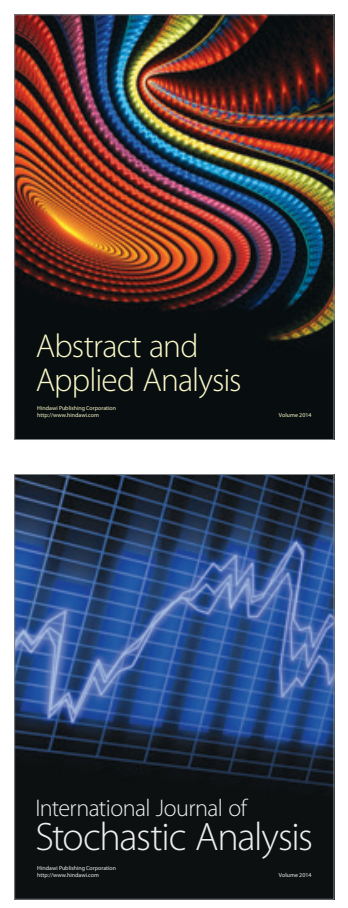

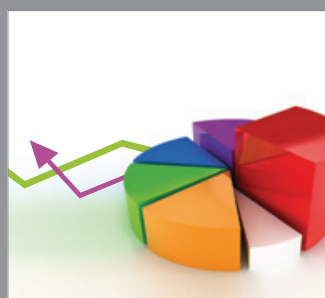

ournal of

Probability and Statistics

Promensencen
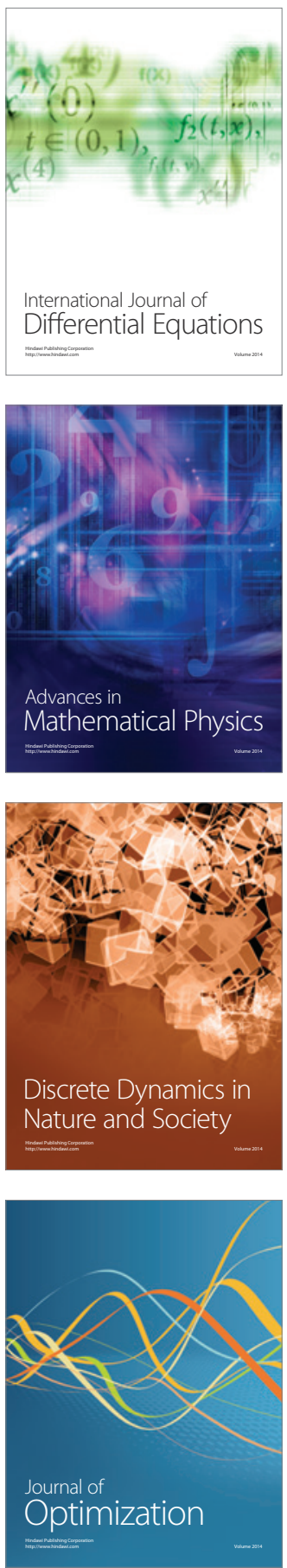